COMMUNICATIONS IN

ANALYSIS AND GEOMETRY

Volume 8, Number 3, 477-515, 2000

\title{
Seiberg-Witten invariants and double covers of 4-manifolds
}

\author{
YongBin RUAN ${ }^{1}$ AND ShUgUang WANG ${ }^{2}$
}

We establish explicit formulas for Seiberg-Witten invariants of (possibly ramified) double covers of smooth 4-manifolds.

\section{Introduction.}

Double covers are an important construction in smooth 4-dimensional manifold theory and had been intensively studied in the pre-gauge theory period. For example, Cappell-Shaneson [2] and Fintushel-Stern [3] used double covers along $\mathbf{R P}^{2}$ in their constructions of 4-manifolds that are homotopic (in fact homeomorphic) but not diffeomorphic to $\mathbf{R P}^{4}$. Akbulut and Kirby [1] obtained the explicit diffeomorphism types for double covers of $S^{4}$ branched along surfaces that can be pushed from a 4 -disk to its boundary $S^{3}$. In a different context, most of the known complex surfaces of general type in the geography problem are constructed as double covers of ruled surfaces through the work of U. Persson, G. Xiao and Z. Chen.

The introduction of Donaldson's gauge theory has revolutionized the study of smooth 4-manifolds. Donaldson theory was particularly useful to study topological constructions in 4-manifolds, such as connected sums and fiber connected sums. One of focal points of Donaldson theory was to find formulas for Donaldson invariants under such constructions, for example, the blow-up formula and the gluing formula for fiber sums over torus. These formulas played a crucial role for the developments of gauge theory and its applications to 4-manifolds. Naturally, we would like to have a formula for Donaldson invariants for the double covers in view of their previous applications.

To obtain such a formula, the second author [23] considered the natural problem how to compare the moduli spaces of anti-self connections on a 4-manifold and its double branched cover. The motivations here are to

\footnotetext{
${ }^{1}$ partially supported by an NSF grant and a Sloan fellowship

${ }^{2}$ partially supported by SRF and Research Board grant of University of Missouri
} 
study connections that have singularities along 2-dimensional surfaces and to construct possibly new "exotic" 4-manifolds from quotient manifolds in connection with real algebraic geometry. This method of studying singular connections has proved to be quite effective in the remarkable work of Kronheimer-Mrowka [11]. However there is much less progress in the goal of relating the Donaldson invariants on a 4-manifold and its branched cover. The difficulty is to do with the fact that in order to have a smooth equivariant moduli space, we need to use an invariant Riemann metric which is "generic" among invariant metrics (the covering manifold has a natural involution action which interchanges the two sheets of the covering map). The same invariant metric is however rarely "generic" among all metrics and hence the associated ordinary moduli space is not necessarily smooth in general. (Hambleton and Lee [6] describe the stratum structure of the ordinary moduli space. See a different treatment in [4].) Regardless of the smoothness, a structure theorem is obtained in [23] for the fixed point set of the ordinary moduli space under the induced involution action, which contains the equivariant moduli space as one component. In the current paper, this kind of structure theorem will be extended and will play an important role.

The recently discovered Seiberg-Witten invariants [26] are much easier to understand than the Donaldson invariants in nearly all the aspects. Complete formulas for Seiberg-Witten invariants of connected sums and fiber connected sums have been obtained. These formulas underline the latest developments in Szabo's examples about irreducible non-symplectic manifolds [22] and Fintushel-Stern's theorems relating Seiberg-Witten invariants to some knot invariants [5]. Seiberg-Witten theory has also brought new light to double cover constructions. For example, in the case of a general type complex surface with free anti-holomorphic involution, it is shown in [24] that the (non-simply connected) quotient manifold has vanishing SeibergWitten invariants but is indecomposable; Kotschick [9] further shows that it is irreducible. In a different direction, the first author [20], [21] developed a new technique (the virtual neighborhood method) in order to deal with the issue that the invariant Riemann metric is not generic among all metrics. One would hope this time to be able to express the relation between Seiberg-Witten invariants on the quotients and the 4-manifolds as branched covers. This is the topic of our paper.

In order to state our theorems in a concrete way, we introduce the setup to be used. Let $p: \widetilde{X} \rightarrow X$ be a smooth double cover branched along an orientable connected surface $\Sigma$. Suppose $\xi$ is a $\operatorname{spin}^{c}$ structure on $X$, 
with determinant line bundle $L$. The virtual dimension of the associated Seiberg-Witten moduli space is

$$
d_{L}=\frac{1}{4}\left(c_{1}(L)^{2}-2 e_{X}-3 s_{X}\right)
$$

where $e_{X}$ and $s_{X}$ are respectively the Euler characteristic and signature of $X$.

First we consider the case that $\Sigma=\emptyset$, namely $p: \widetilde{X} \rightarrow X$ is a unramified double cover. It is clear that there is a well-defined pull-back spin ${ }^{c}$ structure $\widetilde{\xi}$ on $\widetilde{X}$ via the projection $p$. Using the decomposition of the moduli spaces as in [23] and the virtual neighborhood technique of [21], we obtain the following result:

Theorem A. (Theorem 4.2) Suppose that $p: \widetilde{X} \rightarrow X$ is an unramified cover and $\xi$ is a spin ${ }^{c}$ structure of $X$ such that $d_{L}=0, c_{1}(L)$ is nontorsion, and $b_{2}^{+}(\widetilde{X}), b_{2}^{+}(X)>1$. Then the Seiberg-Witten invariants satisfy the following relation:

$$
S W(\widetilde{\xi})=\sum_{\gamma \in \mathcal{K}} S W(\xi \otimes \gamma) \bmod 2
$$

where $\mathcal{K}$ is the set of isomorphic classes of complex line bundles on $X$ which pull back to the trivial bundle on $\widetilde{X}$.

In other words, Theorem A states that modulo $2, S W(\widetilde{\xi})$ is the sum of the Seiberg-Witten invariants of all spin ${ }^{c}$ structures on $X$ that pull back to the $\operatorname{spin}^{c}$ structure $\widetilde{\xi}$. (from [26], there are only a finite number of non-zero terms in the sum.)

Next consider the case $\Sigma \neq \emptyset$. Introduce the adjunction term of $\Sigma$ with respect to $L$ :

$$
J_{L}(\Sigma)=\left|c_{1}(L) \cdot[\Sigma]\right|+[\Sigma]^{2}+e_{\Sigma}
$$

For definiteness, we concentrate on the case of $c_{1}(L) \cdot[\Sigma] \leq 0$. Although $\widetilde{\xi}$ is not a meaningful $\operatorname{spin}^{c}$ structure because of the branched locus $\Sigma$, we can obtain a $\operatorname{spin}^{c}$ structure $\widehat{\xi}$ on $\widetilde{X}$ whose determinant line bundle is $p^{*}(L) \otimes$ $P D[\Sigma]^{-1}$ and whose restriction $\left.\widehat{\xi}\right|_{\widetilde{X} \backslash \widetilde{\Sigma}}$ is isomorphic to the pull-back of $\left.\xi\right|_{X \backslash \Sigma}$ through the projection $p$. Consider the complement

$$
p_{\Sigma}: \widetilde{X} \backslash \widetilde{\Sigma} \rightarrow X \backslash \Sigma
$$

equipped with cylindrical end metrics. Then, $p_{\Sigma}$ is an unramified double cover again and the same argument for proving Theorem A applies to give 
a relation between the relative Seiberg-Witten invariants. Using various gluing results due to Morgan-Szabo-Taubes [15] and Mrowka-Ozsvath-Yu [18], we study the relation between the relative invariant and the invariant of closed manifolds. After some detailed analysis, we obtain

Theorem B. (Theorem 7.14) Let $p: \widetilde{X} \rightarrow X$ be a double cover branched along a surface $\Sigma$ with $[\Sigma]^{2}>0$, and such that $b_{2}^{+}(X)>1, b_{2}^{+}(\widetilde{X})>1$, and $H_{1}(X, \mathbf{Z})$ contains no 2-torsion. Suppose that $\xi$ is a spin ${ }^{c}$ structure on $X$ whose determinant bundle $L$ is such that $c_{1}(L) \cdot P D[\Sigma] \leq 0$, and the virtual dimension and adjunction term both vanish: $d_{L}=J_{L}(\Sigma)=0$. Moreover assume $[\Sigma]^{2}<2 g-2$ and $4 g-4$ is not divisible by $[\Sigma]^{2}$. Then the following equality holds:

$$
S W(\widehat{\xi})=S W(\xi) \bmod 2 .
$$

In the case $[\Sigma]^{2}=0$, we did not obtain a relation between the SeibergWitten invariants of the closed manifolds. However, we have

Theorem C. (Theorem 6.8) Assume $X, \widetilde{X}, \xi, \widetilde{\xi}$ as in Theorem B except that $H_{1}(X, \mathbf{Z})$ is allowed to have 2-torsion. As for $\Sigma$, we assume $[\Sigma]^{2}=0$ and $g>1$. Then

$$
S W(\widehat{\xi})=S W(\xi)+k_{\xi}(X, \Sigma) \bmod 2,
$$

where $k_{\xi}(X, \Sigma)$ is a relative invariant defined in Definition 6.7, which depends on the topology of the embedding $\Sigma \subset X$.

The adjunction equality $J_{L}(\Sigma)=0$ plays a key role in the above theorems, without which the theorems are not valid. The implication of $J_{L}(\Sigma)=0$ is that as far as Seiberg-Witten theory is concerned, the branched cover $p: \widetilde{X} \rightarrow X$ can be treated as complex branched cover along a complex curve. Thus Theorems B and C may be viewed as extensions of the familiar formula relating the canonical bundles of complex branched covers. As a simple application, in the situation of Theorem B, if $X$ is a complex surface of general type then any smooth double cover $\widetilde{X}$ always has a Seiberg-Witten basic class.

The reader probably notices that we left out the case $[\Sigma]^{2}=0$ and $g=0,1$. We remark that Theorem $\mathrm{C}$ fails in this case due to the more complicated gluing formula. We leave a more complete study to a future project.

The paper is organized as follows: In Section 2 we will use the virtual neighborhood method to prove a relation between a Donaldson type invariant and the invariant counting the fixed points of an involution. In Section 3 , 
we prove the structure theorem of Seiberg-Witten theory with a $Z_{2}$-action. We apply results of Sections 2 and 3 to prove Theorem A in Section 4. In Section 5, we collect some basic topological properties for branched covers, by combining which with the study of relative invariants in details we prove Theorem C in Section 6 and Theorem B in Section 7.

Convention. As it is commonly used now, we will abbreviate the word Seiberg-Witten as SW in this paper without further remark.

\section{A comparison theorem.}

Recall that the famous Lefschetz fixed point formula relates the information of fixed points to that of the total space. Our result in this section can be regarded as a generalization of the Lefschetz formula. Suppose that $\mathcal{B}$ is a smooth oriented Hilbert manifold and $\mathcal{F}$ is a smooth oriented Hilbert bundle. Let $F: \mathcal{B} \rightarrow \mathcal{F}$ be a Fredholm section, namely $F$ is represented by Fredholm maps in local trivilizations of $\mathcal{F}$. Throughout the section we assume that the moduli space $\mathcal{M}=F^{-1}(0)$ is compact but not necessarily smooth. Let $\tau$ be an orientation preserving involution acting on $\mathcal{B}, \mathcal{F}$ and commuting with the projection $\pi: \mathcal{F} \rightarrow \mathcal{B}$. Let $\mathcal{M}_{f} \subset \mathcal{M}$ be the fixed point set. For any $x \in \mathcal{M}_{f}, \tau$ acts on ker $D F_{x}$ and coker $D F_{x}$. The induced action of $\tau$ on these spaces has eigenvalues \pm 1 as $\tau$ is an involution. Let $\operatorname{ker}^{ \pm} D F_{x}, \operatorname{coker}^{ \pm} D F_{x}$ be the \pm 1 eigenspaces. The virtual dimension of $\mathcal{M}_{f}$ is

$$
\operatorname{dim} \operatorname{ker}^{+} D F-\operatorname{dim} \operatorname{coker}^{+} D F \text {; }
$$

an $x \in \mathcal{M}_{f}$ is a smooth point of $\mathcal{M}_{f}$ if and only if coker ${ }^{+} D F_{x}=0$. In [21], the first author outlined a so-called virtual neighborhood method to extract invariants $\Phi_{f}, \Phi$ from $\mathcal{M}_{f}$ and $\mathcal{M}$ respectively. The virtual neighborhood method can also be used to compare $\Phi_{f}$ and $\Phi$. This is the purpose of this section. Although we are concerned only with Seiberg-Witten equations in this paper, the result in this section holds in general. Therefore, we shall work on the general framework of Hilbert manifolds and Fredholm maps. For a technical reason, we assume that the virtual dimensions of both $\mathcal{M}_{f}$ and $\mathcal{M}$ are zero.

In cases such as Seiberg-Witten equations, we can actually choose a generic $\tau$-invariant Riemann metric and a generic $\tau$-invariant perturbation such that $\mathcal{M}_{f}$ is a smooth manifold (but $\mathcal{M}$ may not be smooth). This is because the usual transversality argument still works in the equivariant set 
up. In other words we can assume $\operatorname{coker}^{+} D F_{x}=0$ for any $x \in \mathcal{M}_{f}$. On the other hand, the virtual dimension of $\mathcal{M}_{f}$ is zero. Therefore, $\mathcal{M}_{f}$ is a set of discrete points and $\Phi_{f}=\# \mathcal{M}_{f} \bmod 2$.

Theorem 2.2. Suppose that the virtual dimensions of $\mathcal{M}_{f}$ and $\mathcal{M}$ are zero and $\mathcal{M}_{f}$ is a smooth manifold. Then we have

$$
\Phi_{f}=\Phi \bmod 2 .
$$

Proof. Suppose that $x \in \mathcal{M}_{f}$. By the assumptions,

$$
\operatorname{ker}^{+} D F_{x}=\operatorname{coker}^{+} D F_{x}=0
$$

and

$$
\operatorname{dim} \operatorname{ker}^{-} D F_{x}=\operatorname{dim} \operatorname{coker}^{-} D F_{x} .
$$

The first step is to add a local equivariant perturbation $\sigma$ such that $x$ is a smooth point of $\mathcal{M}_{\sigma}=(F+\sigma)^{-1}(0)$. Locally, we can view $F$ as a map $T_{x} \mathcal{B} \rightarrow \mathcal{F}_{x}\left(\mathcal{F}_{x}\right.$ is the fiber over $\left.x\right)$. Recall that we have a local Kuranishi model of $F$ as follow: There are orthogonal splittings

$$
\begin{aligned}
T_{x} \mathcal{B} & =\operatorname{ker} D F_{x} \oplus X, \\
\mathcal{F}_{x} & =\operatorname{im} D F_{x} \oplus \operatorname{coker} D F_{x}
\end{aligned}
$$

and a map

$$
\phi: X \rightarrow \operatorname{coker} D F_{x}
$$

such that there is a change of local coordinates $\chi: T_{x} \mathcal{B} \rightarrow T_{x} \mathcal{B}$ with the properties

$$
F \circ \chi^{-1}=D F_{x}+\phi
$$

and $\phi(0)=0,(d \phi)_{0}=0$. Furthermore, $\chi, \phi, X$ are $\tau$-equivariant.

By (2.5), $\operatorname{ker} D F_{x}=\operatorname{ker}^{-} D F_{x}$ and coker $D F_{x}=\operatorname{coker}^{-} D F_{x}$. Furthermore,

$$
\operatorname{dim} \operatorname{ker} D F_{x}=\operatorname{dim} \operatorname{coker} D F_{x} .
$$

Therefore, there is a $\tau$-equivariant isomorphism

$$
\phi^{\prime}: \operatorname{ker} D F_{x} \rightarrow \operatorname{coker} D F_{x} .
$$


Let $\beta: T_{x} \mathcal{B} \rightarrow \mathbf{R}$ be a $\tau$-equivariant cut-off such that $\beta=1$ in a small ball around the origin and vanishes outside a slightly larger ball. Furthermore, we assume that $\operatorname{supp}(\beta) \cap \mathcal{M}_{f}=\{x\}$. Let $\epsilon>0$ and let $\sigma=\epsilon \beta \phi^{\prime}$. Consider the perturbed equation

$$
F_{\sigma} \circ \chi^{-1}=T+\phi+\sigma .
$$

Clearly, $F_{\sigma}$ is $\tau$-equivariant and $F_{\sigma}(x)=0$. Moreover,

$$
\left(D F_{\sigma}\right)_{0} \cong T+\epsilon \phi^{\prime}
$$

is an isomorphism. So, $x$ is a smooth point of $F_{\sigma}$. Therefore, to simplify notation, we can assume that any point $x \in \mathcal{M}_{f}$ is a smooth point of $\mathcal{M}$. Hence, we have a decomposition

$$
\mathcal{M}=\mathcal{M}_{f} \cup \mathcal{M}_{r}
$$

where $\mathcal{M}_{r}$ is compact and disjoint from $\mathcal{M}_{f}$.

Next, we use the virtual neighborhood method to compare $\Phi_{f}$ and $\Phi$. Let us give a brief outline of the virtual neighborhood method [21]. First, we find finitely many sections $s_{1}, \cdots, s_{k}$ defined over a neighborhood $\mathcal{U}$ of $\mathcal{M}$ such that

$$
D F+\sum t_{i} s_{i}: T \mathcal{B} \times \mathbf{R}^{k} \rightarrow \mathcal{F}
$$

is surjective on $\mathcal{M}$. Then, we change our equation to

$$
F_{v}=F+\sum_{i} t_{i} s_{i}: \mathcal{U} \times \mathbf{R}^{k} \rightarrow \mathcal{F} .
$$

By the construction, $\mathcal{M} \times\{0\} \subset F_{v}^{-1}(0)$ and its linearization $\delta F_{v}$ is surjective on $\mathcal{M} \times\{0\}$. Hence, we can assume that it is surjective on $\mathcal{U} \times \mathbf{R}^{k}$. Therefore,

$$
U=F_{v}^{-1}(0) \subset \mathcal{U} \times \mathbf{R}^{k}
$$

is a finite dimensional smooth manifold and $\mathcal{M} \times\{0\} \subset U$. Let

$$
S: U \rightarrow \mathbf{R}^{k}
$$

be defined by $S\left(A, t_{i}\right)=t_{i}$. Then $S^{-1}(0)=\mathcal{M} \times\{0\}$. Since $\mathcal{M}$ is compact, we can assume that $S$ is proper by shrinking the neighborhood. Then, from the infinite dimensional triple $(\mathcal{B}, \mathcal{F}, F)$, we construct a finite dimensional triple $\left(U, \mathbf{R}^{k}, S\right)$ called a virtual neighborhood. To define a topological invariant, one just applies the finite dimensional technique to $\left(U, \mathbf{R}^{k}, S\right)$. 
In our case, we need to construct a $\tau$-equivariant virtual neighborhood. Since $F$ is already transverse at $\mathcal{M}_{f}$, we don't have to do anything there. For any $x \in \mathcal{M}_{r}$, extend a basis $u_{1}, \cdots, u_{i_{x}}$ of coker $D F_{x}$ to be sections (still denoted by $u_{i}$ ) supported in a neighborhood of $x \in \mathcal{B}$. We choose $u_{i}$ such that

$$
\tau\left(\operatorname{supp}\left(u_{i}\right)\right) \cap \operatorname{supp}\left(u_{i}\right)=\emptyset
$$

and

$$
\left(\operatorname{supp}\left(u_{i}\right) \cup \operatorname{supp}\left(\tau\left(u_{i}\right)\right)\right) \cap \mathcal{M}_{f}=\emptyset .
$$

Then, we can construct $\tau$-equivariant sections by

$$
s_{i}=u_{i}+\tau^{*} u_{i} .
$$

Clearly, $\left.s_{i}\right|_{\operatorname{supp}\left(u_{i}\right)}=u_{i}$. By continuity, $\left\{u_{i}\right\}$ and hence $\left\{s_{i}\right\}$ generate coker $D F_{y}$ for all $y$ in some neighborhood $U_{x}$ of $x$. Choose finitely many $U_{x}$ s to cover $\mathcal{M}_{r}$ and such that $U_{x} \cap M_{f}=\emptyset$. Enumerate all the corresponding sections as $s_{1}, \cdots, s_{k}$. Then,

(i) $s_{i}$ is $\tau$-equivariant,

(ii) $\operatorname{supp}\left(s_{i}\right) \cap \mathcal{M}_{f}=\emptyset$, and

(iii) $s_{1}, \cdots, s_{k}$ generate coker $D F_{y}$ for $y \in \mathcal{M}$.

These mean that $D F_{y}+\sum_{i} t_{i} s_{i}$ satisfies the requirement of the virtual neighborhood construction and we can construct a virtual neighborhood $\left(U, \mathbf{R}^{k}, S\right)$. Note that $F_{v}: \mathcal{B} \times \mathbf{R}^{k} \times \mathcal{F}$ is $\tau$-equivariant with a trivial action on $\mathbf{R}^{k}$. Therefore, $\tau$ acts on $U$ and $S$ is $\tau$-equivariant. One can check that the fixed points set $U_{f}$ of $U$ is

$$
U_{f}=U \cap\left(\mathcal{M}_{f} \times \mathbf{R}^{k}\right) .
$$

Since $\tau$ acts on $\mathbf{R}^{k}$ trivially, we can choose a $\tau$-invariant regular value $y$ of $S$ close to 0 . Moreover,

$$
\Phi=\# S^{-1}(y) \bmod 2 .
$$

Clearly, $\tau$ acts on $S^{-1}(y)$ and

$$
S^{-1}(y)=S^{-1}(y)_{f} \cup S^{-1}(y)_{r},
$$

where $S^{-1}(y)_{f}$ is the fixed points set and $S^{-1}(y)_{r}$ is the rest. Obviously,

$$
\Phi=\# S^{-1}(y)_{f} \bmod 2 .
$$


By (2.12),

$$
S^{-1}(y)_{f}=S^{-1}(y) \cap\left(\mathcal{M}_{f} \times \mathbf{R}^{k}\right)=\mathcal{M}_{f} \times\{y\} .
$$

Therefore, $\Phi=\Phi_{f} \bmod 2$, and we finish the proof.

Remark. For the fixed point set of a $\mathbf{Z}_{n}$ action, $n>2$, one can also construct an equivariant virtual neighborhood. However, the action on $\mathbf{R}^{k}$ is not trivial.

\section{Seiberg-Witten theory with a $Z_{2}$-action.}

In the literature on gauge theory with a group action, the typical situation is to compare the equivariant moduli space on a manifold with the moduli space on the quotient manifold under a group action. In this section we undertake a different direction of investigation: we will compare the ordinary moduli space on a manifold, via the fixed moduli space, with the moduli space on the quotient manifold.

Let $p: \widetilde{Y} \rightarrow Y$ be a smooth unramified double cover of Riemannian 4manifolds (not necessarily compact) and $\sigma: \widetilde{Y} \rightarrow \widetilde{Y}$ the covering involution. The metric $\widetilde{h}$ on $\widetilde{Y}$ is assumed to be the pull-back of a metric $h$ on $Y$. A $\operatorname{spin}^{c}$ structure will be viewed either as a $\operatorname{Spin}^{c}(4)$-principal bundle or as a pair of $U(2)$-vector bundles satisfying the usual conditions. Specifically let a $\operatorname{spin}^{c}$ structure $\xi$ on $Y$ be given by a $\operatorname{Spin}^{c}(4)$-bundle $P \rightarrow Y$ with determinant line bundle $L$ and spinor bundles $W^{ \pm}$. Denote their pull-backs on $\widetilde{Y}$ respectively by $\widetilde{\xi}, \widetilde{P}, \widetilde{L}$ and $\widetilde{W}^{ \pm}$, through the projection $p: \widetilde{Y} \rightarrow Y$.

Consider a lifting $\tau$ of $\sigma_{*}$ :

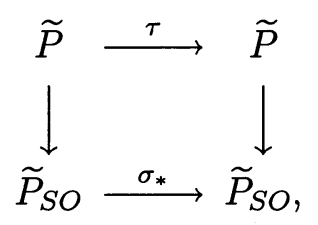

where $\widetilde{P}_{S O}$ is the frame bundle of $\tilde{Y}$. Let $\hat{\tau}=\operatorname{det} \tau$, then we also have the commutative diagram

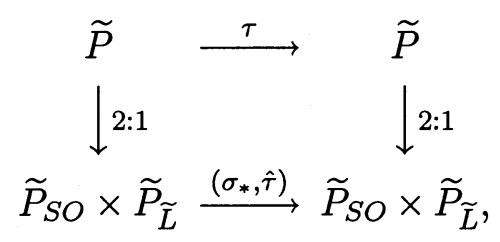


where $\widetilde{P}_{\widetilde{L}}$ is the principal $U(1)$-bundle associated to $\widetilde{L}$. It is clear that $\tau$ is determined by $\sigma_{*}$ and $\hat{\tau}$ up to \pm signs.

As usual let $\mathcal{A}(\widetilde{L}), \Gamma\left(\widetilde{W}^{+}\right)$respectively denote the space of connections on $\widetilde{L}$ and the space of sections on $\widetilde{W}^{+}$. (Throughout this section, we suppress without harm the usual Sobolev orders for all spaces involved.)

Definition 3.3. If $(A, \phi) \in \mathcal{A}(\widetilde{L}) \times \Gamma\left(\widetilde{W}^{+}\right)$, then define

$$
\tau^{*}(A, \phi)=\left(\hat{\tau}^{*} A,\left(\tau^{-1}\right)^{*} \phi\right)
$$

where $\hat{\tau}^{*} A$ is given by

$$
D_{\hat{\tau}^{*} A}(s)=\hat{\tau}\left(D_{A}\left(\hat{\tau}^{-1} \circ s \circ \sigma\right)\right)
$$

for $s \in \Gamma(\widetilde{L})$ and $\left(\tau^{-1}\right)^{*} \phi=\tau^{-1} \circ \phi \circ \sigma$.

If $g$ is a gauge transformation, then define $\tau^{*} g=\tau \circ g \circ \tau^{-1}$. (Recall that a gauge transformation is an automorphism on $\widetilde{P}$ which covers the identity on $\widetilde{P}_{S O}$ in (3.1).)

Observe that $\tau \circ g \circ \tau^{-1}=g_{0} \circ \sigma$, where $g_{0}: \tilde{Y} \rightarrow S^{1}$ is the map whose multiplication gives rise to $g$; thus the $\tau^{*}$ action on a gauge is uniquely determined by that of $\sigma$. Under the same veil, since any two liftings in (3.1) differ by a gauge transformation, the induced action of $\tau^{*}$ on the configuration space $\mathcal{B}(\widetilde{\xi})=\mathcal{A}(\widetilde{L}) \times \Gamma\left(\widetilde{W}^{+}\right) / \mathcal{G}$ is independent of the choice of $\tau$, where $\mathcal{G}$ is the space of gauge transformations. Let $\mathcal{B}(\widetilde{\xi})^{\tau}$ be the fixed point set of $\tau^{*}: \mathcal{B}(\widetilde{\xi}) \rightarrow \mathcal{B}(\widetilde{\xi})$

Using the action $\tau^{*}$ on $\mathcal{A}(\widetilde{L}) \times \Gamma\left(\widetilde{W}^{+}\right)$, we set

$$
\mathcal{B}(\widetilde{\xi})_{\tau}=\frac{\left\{\tau^{*} \text {-invariant pairs }(A, \phi)\right\}}{\left\{\tau^{*} \text {-invariant gauge transformations }\right\}}
$$

Note that unlike $\mathcal{B}(\widetilde{\xi})^{\tau}$, the set $\mathcal{B}(\widetilde{\xi})_{\tau}$ does depend on the choice of $\tau$. It is straightforward to check that the map

$$
\begin{aligned}
\mathcal{A}(\widetilde{L}) \times \Gamma\left(\widetilde{W}^{+}\right) & \rightarrow \mathcal{B}(\widetilde{\xi}) \\
(A, \phi) & \mapsto[A, \phi]
\end{aligned}
$$

induces injective maps $\mathcal{B}(\widetilde{\xi})_{\tau}^{*} \rightarrow \mathcal{B}(\widetilde{\xi})^{* \tau}$ (as usual * denotes irreducible pairs $(A, \phi)$, i.e. $\phi \neq 0)$. We will regard $\mathcal{B}(\widetilde{\xi})_{\tau}^{*}$ as subset of $\mathcal{B}(\widetilde{\xi})^{* \tau}$ by identifying it with its image. 
Consider also the SW moduli space $M(\widetilde{\xi})=M(\widetilde{\xi}, \widetilde{h}, \widetilde{\mu}) \subset \mathcal{B}(\widetilde{\xi})$, namely the solution space, up to gauge transformations, of the perturbed SW equations for $(A, \phi) \in \mathcal{A}(\widetilde{L}) \times \Gamma\left(\widetilde{W}^{+}\right)$:

$$
\begin{aligned}
\not D_{A}(\phi) & =0 \\
F_{A}^{+} & =q(\phi)+i \tilde{\mu},
\end{aligned}
$$

where $q(\phi)=\phi \otimes \phi^{*}-\frac{|\phi|^{2}}{2} \mathrm{Id}$, and $\widetilde{\mu} \in \Omega_{+}^{2}(\tilde{Y})$ and $\widetilde{h}$ are assumed to be invariant under $\sigma$. By restricting the relevant actions, we obtain the fixed moduli space $M(\widetilde{\xi})^{\tau} \subset \mathcal{B}(\widetilde{\xi})^{\tau}$ and the equivariant moduli space $M(\widetilde{\xi})_{\tau} \subset$ $\mathcal{B}(\widetilde{\xi})_{\tau}$. Again while $M(\widetilde{\xi})^{* \tau}$ is independent of $\tau$, the subset $M(\widetilde{\xi})_{\tau}^{*}$ does depend on the choice of the lifting $\tau$.

Let $\gamma$ be a complex line bundle on $Y$. The $U(2)$-bundles $W^{ \pm} \otimes \gamma$ then give a twisted $\operatorname{spin}^{c}$ structure $\xi \otimes \gamma$ on $Y$. Let $M(\xi)=M(\xi, h, \mu)$ and $M(\xi \otimes \gamma)=M(\xi \otimes \gamma, h, \mu)$ be the SW moduli spaces on $Y$ with the indicated $\operatorname{spin}^{c}$ structures, metric and perturbation. We now have the structure of the fixed moduli space and the relation with moduli spaces on the quotient, which is a key step in this paper. For later applications we emphasize that $Y$ and $\widetilde{Y}$ are allowed to be non-compact here. Write $x^{\sigma}=\sigma(x), \forall x \in \tilde{Y}$ for convenience.

Theorem 3.8. Suppose $p: \widetilde{Y} \rightarrow Y$ is an unramified double cover, $\xi$ is a spin $^{c}$ structure on $Y$ and $\widetilde{\xi}$ is the pull-back on $\widetilde{Y}$. Let now $\tau$ be the natural lifting on $\widetilde{\xi}$ given as the pull back of the identity isomorphism on $\xi$.

(1) There is a natural disjoint decomposition

$$
M(\widetilde{\xi})^{* \tau}=\coprod_{[k] \in K} M(\widetilde{\xi})_{k \tau}^{*}
$$

indexed by the set $K$ of equivalence classes of maps $k: \widetilde{Y} \rightarrow S^{1}$ satisfying $k\left(x^{\sigma}\right) k(x)=1$ for all $x \in \widetilde{Y}$, where $k \sim k^{\prime}$ if and only if $g\left(x^{\sigma}\right) k(x)=g(x) k^{\prime}(x)$ for some map $g: \widetilde{Y} \rightarrow S^{1}$. Moreover, under the natural multiplication, $K$ is a group consisting of 2-torsion only; in particular the union in (3.9) is finite.

(2) There is a natural isomorphism $K \rightarrow \mathcal{K}$, where $\mathcal{K}$ is the set of isomorphism classes of line bundles on $Y$ that pull back to the trivial bundle on $\tilde{Y}$ via the projection $p: \widetilde{Y} \rightarrow Y$. Furthermore for each $[k] \in K$, there is a natural homeomorphism $M(\widetilde{\xi})_{k \tau} \rightarrow M\left(\xi \otimes \gamma_{k}\right)^{*}$, 
where $\gamma_{k} \in \mathcal{K}$ is the line bundle associated to $k$. Consequently we have a natural homeomorphism

$$
M(\widetilde{\xi})^{* \tau} \longrightarrow \coprod_{[\gamma] \in \mathcal{K}} M(\xi \otimes \gamma)^{*}
$$

Proof. (1) Let $[A, \phi] \in M(\widetilde{\xi})^{* \tau}$. We first show that there exists a $k: \widetilde{Y} \rightarrow S^{1}$ satisfying $k\left(x^{\sigma}\right) k(x)=1$ for all $x \in \widetilde{Y}$, such that $[A, \phi] \in M(\widetilde{\xi})_{k \tau}^{*}$. Indeed since $[A, \phi] \in M(\widetilde{\xi})^{* \tau}$, there is a gauge $g$ such that $\tau^{*}(A, \phi)=g^{*}(A, \phi)$; so $\left(\tau \circ g^{-1}\right)^{*}(A, \phi)=(A, \phi)$. Let $\tau^{\prime}=\tau \circ g^{-1}$ then $[A, \phi] \in M(\tilde{Y})_{\tau^{\prime}}^{*}$ and ${\tau^{\prime}}^{2}$ is a gauge fixing $(A, \phi)$; by irreducibility of $(A, \phi)$ one has ${\tau^{\prime}}^{2}=1$. Write $\tau^{\prime}=k \tau$ for a unique map $k: \widetilde{Y} \rightarrow S^{1}$; then ${\tau^{\prime}}^{2}=1$ implies $k\left(x^{\sigma}\right) k(x)=1$. So we have shown $M(\widetilde{\xi})^{* \tau}=\cup_{[k] \in K} M(\widetilde{\xi})_{k \tau}^{*}$.

Using the irreducibility alone, a similar argument as above also shows that $M(\widetilde{\xi})_{k \tau}^{*}$ and $M(\widetilde{\xi})_{k^{\prime} \tau}^{*}$ are either identical or disjoint, and they are identical if and only if $k \sim k^{\prime}$. Moreover for any $k \in K, k\left(x^{\sigma}\right) k(x)=1$ implies $g\left(x^{\sigma}\right) k(x)^{2}=g(x)$ with $g(x)=k(x)$, namely $k^{2} \sim 1$. So $k$ is either trivial or a 2 -torsion.

(2) For any $k: \widetilde{Y} \rightarrow S^{1}$ satisfying $k\left(x^{\sigma}\right) k(x)=1$, the quotient manifold $Q=\left(\widetilde{Y} \times S^{1}\right) /(x, r) \sim\left(x^{\sigma}, k(x) r\right)$ is well-defined. By projecting to the first factor one gets an $S^{1}$-principal bundle $\gamma_{k}=Q \rightarrow Y$, and one can check easily that this gives rise to an isomorphism $K \rightarrow \mathcal{K}$. For instance to check. the surjectivity, let $\gamma \rightarrow Y$ be an $S^{1}$ - bundle with trivial pull-back $p^{*} \gamma \rightarrow \widetilde{Y}$. Choose any global trivilization $s: \widetilde{Y} \rightarrow p^{*} \gamma$ and define $k: \widetilde{Y} \rightarrow S^{1}$ by requiring $p(s(x))=k(x) p\left(s\left(x^{\sigma}\right)\right) \in \gamma_{p(x)}$. Then $k$ is independent of the choice of $s$ and $\gamma=\gamma_{k}$.

Finally to construct the homeomorphism $M(\widetilde{\xi})_{k \tau}^{*} \rightarrow M\left(\xi \otimes \gamma_{k}\right)^{*}$, consider the $U(2)$-bundles on $\widetilde{Y}, \widetilde{W}_{c}^{ \pm}=\widetilde{W}^{ \pm} \otimes \underline{\mathbf{C}}$, where $\underline{\mathbf{C}}$ is the trivial line bundle. Let $\rho: \widetilde{W}_{c}^{ \pm} \rightarrow \widetilde{W}_{c}^{ \pm}$be the action given as

$$
\rho(u \otimes v)=\tau(u) \otimes(k v)
$$

$\rho$ should be understood as an order-two lifting of $\sigma$. By pull-back, $M\left(\widetilde{\xi}_{c}\right)_{\rho}^{*}$ is homeomorphic to the irreducible moduli space on $Y$ with $\operatorname{spin}^{c}$ structure

$$
\widetilde{W}_{c}^{ \pm} / \rho=W^{ \pm} \otimes(\underline{\mathbf{C}} / k \cdot)=W^{ \pm} \otimes \gamma_{k},
$$

namely $M\left(\widetilde{\xi}_{c}\right)_{\rho}^{*}$ is homeomorphic to $M\left(\xi \otimes \gamma_{k}\right)^{*}$.

On the other hand $\rho=(k \tau) \otimes \mathrm{Id}$, so we have the 1-1 correspondence $\{(k \tau)$-invariant $\mathrm{SW}$ solutions $\} \longleftrightarrow\{\rho$-invariant $\mathrm{SW}$ solutions $\}$ 
and it follows that $M(\widetilde{\xi})_{k \tau}^{*}$ is homeomorphic to $M\left(\widetilde{\xi}_{c}\right)_{\rho}^{*}$. Combining with the previous paragraph, we have that $M(\widetilde{\xi})_{k \tau}^{*}$ is homeomorphic to $M\left(\xi \otimes \gamma_{k}\right)^{*}$.

Remark. (1) The complex line bundle $\lambda \rightarrow Y$ associated to the double cover $\tilde{Y} \rightarrow Y$ (a $\mathbf{Z}_{2}$-bundle) obviously belongs to $\mathcal{K}$ which in general is larger than $\{[\lambda], 0\}$. Note that $\lambda$ may be trivial, although the real line bundle $\alpha \rightarrow Y$ associated to $\widetilde{Y} \rightarrow Y$ is never trivial from the connectedness of $\tilde{Y}$ ( $\lambda$ is the complexification of $\alpha$ ).

(2) Chern class yields the identification $\mathcal{K}=\operatorname{ker}\left\{p^{*}: H^{2}(Y, \mathbf{Z}) \rightarrow\right.$ $\left.H^{2}(\widetilde{Y}, \mathbf{Z})\right\}$, which gives another way to index the unions in $(3.9)$ and (3.10). The Gysin sequence of the $S^{0}$-bundle $\tilde{Y} \rightarrow Y$ shows that $\operatorname{ker} p^{*}$ is the image of the map $\wedge e: H^{1}(Y) \rightarrow H^{2}(Y)$, where $e \in H^{1}(Y)$ is the Euler class of the sphere bundle. Since $2 e=0$, one sees again that $\operatorname{ker} p^{*}$ consists of some 2-torsion plus the trivial element.

(3) The theorem remains to be true if $M(\widetilde{\xi})^{*}$ is replaced by $\mathcal{B}(\widetilde{\xi})^{*}$ throughout. In fact since only the irreducibility is used in the proof, neither the solution space $M(\widetilde{\xi})^{*}$ nor the dimension of the manifold $\widetilde{Y}$ matter for the conclusion of the theorem.

\section{SW invariants on unramified covers.}

Based on the structure theorem in the previous section, we compare here the corresponding smooth topological invariants of the moduli spaces. An initial attempt in this direction was made in [23] for instanton moduli spaces and the Donaldson invariants, which provides some rudiments for our discussions below. In connection with group actions Seiberg-Witten invariants are easier to handle because of the clearer display of Seiberg-Witten basic classes in the quotient map. Combined with the virtual neighborhood method, this makes it possible for us to get a better understanding of the relation between the invariants.

We continue using the set-up of Section 3 and confine ourselves to the special case that $Y$ and $\widetilde{Y}$ are both compact. In addition we assume $b_{2}^{+}(Y)>$ $1, b_{2}^{+}(\widetilde{Y})>1$ so that the $\mathrm{SW}$ invariants are well-defined on $\widetilde{Y}, Y$. (The assumption $b_{2}^{+}(\tilde{Y})>1$ may be surplus in some cases e.g. when $b_{1}(Y)=0$, as we have $b_{2}^{+}(\tilde{Y})=1+b_{1}(\tilde{Y})+2 b_{2}^{+}(Y)-2 b_{1}(Y)$ from the usual Euler characteristic and signature formulas for the double cover $\widetilde{Y} \rightarrow Y$.) 
Lemma 4.1. Suppose $c_{1}(L)$ is not a torsion class. Then there is a generic metric $h$ on $Y$ such that with respect to $h, \widetilde{h}=p^{*} h$ respectively, the spin ${ }^{c}$ structures $\xi \otimes \gamma, \gamma \in \mathcal{K}$, and $\widetilde{\xi}$ all admit no reducible $S W$ solutions with perturbations $\mu=\widetilde{\mu}=0$.

Proof. Since $c_{1}(L)$ is not torsion, the class $c_{1}\left(L^{2}\right)$ is not torsion either (they are equivalent statements). By the usual transversality argument, there is a generic metric $h$ on $Y$ such that the class $c_{1}\left(L^{2}\right)$ (more precisely its image in $H^{2}(Y, \mathbf{R})$, the same below) is not represented by any $h$-ASD (=anti self dual) harmonic 2-form. We claim that $\widetilde{\xi}$ admits no reducible un-perturbed $\mathrm{SW}$ solutions with respect to the pull-back metric $\widetilde{h}$. Since otherwise, $c_{1}(\widetilde{L})$ is represented by an $\widetilde{h}$-ASD harmonic form $\alpha$, hence $c_{1}\left(\widetilde{L}^{2}\right)$ is represented by the $\sigma$-invariant $\widetilde{h}$-ASD form $\bar{\alpha}=\alpha+\sigma^{*} \alpha$. Thus $c_{1}\left(L^{2}\right)$ is represented by the pushing down of $\bar{\alpha}$, which is an $h$-ASD harmonic form. This contradicts our choice of $h$.

Any reducible solution on a $\xi \otimes \gamma$ would pull back to a reducible solution on $p^{*} \xi \otimes p^{*} \gamma=\widetilde{\xi}$. Thus the non-existence of reducibles on $\widetilde{\xi}$ implies that on $\xi \otimes \gamma$ for any $\gamma \in \mathcal{K}$.

More directly, reducible solutions can be avoided under the stronger assumption that $c_{1}(L)^{2}>0$, which implies $c_{1}(\widetilde{L})^{2}=2 c_{1}(L)^{2}>0$, and hence $\xi \otimes \gamma$ and $\widetilde{\xi}$ do not admit reducible solutions by a simple argument as in [24].

Our main result in the section is the following:

Theorem 4.2. Let $\xi$ be a spin ${ }^{c}$ structure on $Y$ and $\widetilde{\xi}=p^{*} \xi$ be its pull-back on $\tilde{Y}$. Suppose that $c_{1}(L)$ is not torsion and the virtual dimension of the moduli space associated to $\xi$ is zero. Then we have the following relation among the $S W$ invariants:

$$
S W(\widetilde{\xi})=\sum_{\gamma \in \mathcal{K}} S W(\xi \otimes \sim) \bmod 2
$$

where $\mathcal{K}$ is isomorphism classes of complex line bundles on $Y$ that pull back to the trivial bundle on $\tilde{Y}$.

Proof. For virtual dimensions, we have $\operatorname{dim} M(\widetilde{\xi})=2 \operatorname{dim} M(\xi)=0$ and $\operatorname{dim} M(\xi \otimes \gamma)=\frac{1}{2} \operatorname{dim} M(\widetilde{\xi})=0$. Thus the SW invariants involved are all obtained by counting the moduli spaces in a suitable way. 
Fix a generic metric $h$ on $X$ as in Lemma 4.1, so that

$$
M(\widetilde{\xi}), M(\xi \otimes \gamma), \gamma \in \mathcal{K}
$$

all do not contain reducible solutions for any small perturbations $\mu$ and $\tilde{\mu}=p^{*} \mu$.

By Theorem 3.8, the fixed moduli space $M(\widetilde{\xi})^{\tau}=\bigsqcup_{k \in K} M(\widetilde{\xi})_{k \tau}^{*}$ is homeomorphic to

$$
\coprod_{\gamma \in \mathcal{K}} M(\xi \otimes \gamma)
$$

The compactness theorem in [14] implies that $M(\xi \otimes \gamma)$ s are all compact; thus $M(\widetilde{\xi})^{\tau}$ is also a compact space. By the usual transversality argument applied to our equivariant moduli spaces $M(\widetilde{\xi})_{k \tau}^{*}$, we can choose a small generic $\sigma$ invariant perturbation $\tilde{\mu}$ such that $M(\widetilde{\xi})^{\tau}$ is non-degenerate everywhere and so has the smooth discrete topology. From Theorem 2.2, its associated SW invariant $S W_{f}(\widetilde{\xi})=\# M(\widetilde{\xi})^{\tau}$ is subject to the relation

$$
S W_{f}(\widetilde{\xi})=S W(\widetilde{\xi}) \bmod 2 .
$$

Under the same perturbation, $M(\widetilde{\xi})^{\tau}$ is now diffeomorphic to the union in (4.4) and hence

$$
S W_{f}(\widetilde{\xi})=\sum_{\gamma \in \mathcal{K}} S W(\xi \otimes \gamma) .
$$

Together with (4.5), we arrive at the desired result.

Remark. There are examples of unramified double covers $\widetilde{Y} \rightarrow Y$ in which $\tilde{Y}$ has a $\operatorname{spin}^{c}$ structure with non-trivial SW invariant and $Y$ has vanishing SW invariants for all $\operatorname{spin}^{c}$ structures, e.g., take $Y$ to be the quotient of a free anti-holomorphic involution on a complex surface $\tilde{Y}$ (see [24]). Thus in general, not all basic classes on $\widetilde{Y}$ are pull-backs from $Y$.

Examples. (1) Suppose $Y$ is a minimal complex surface of general type with $b_{2}^{+}(Y)>1$ and $\xi$ is the natural spin $^{c}$ structure associated. Suppose also there exists a smooth unramified double cover $p: \widetilde{Y} \rightarrow Y$. Then $\widetilde{Y}$ inherits a structure of a minimal complex surface of general type and $\widetilde{\xi}=p^{*} \xi$ is associated spin $^{c}$ structure. According to Witten [26] for example, we have $S W(\widetilde{\xi})=1, S W(\xi)=1$ and $S W(\xi \otimes \gamma)=0$ for non-trivial $\gamma$, with which our theorem above is consistent. Indeed Theorem 4.2 may be viewed as some sort of generalization to arbitrary smooth double covers $p: \widetilde{Y} \rightarrow Y$. 
(2) (Kotschick-Morgan-Taubes [10]) Let $N$ be a smooth 4-manifold such that $b_{1}(N)=b_{2}^{+}(N)=0$ and $N$ has an unramified double cover. Then for any smooth 4-manifold $Y_{0}$ whose $\mathrm{SW}$ invariant is non-trivial, it is shown in [10] that $Y=Y_{0} \# N$ has a non-trivial invariant $S W(\xi)$ but the double cover $\widetilde{Y}$ of $Y$ is a connected sum and so has trivial invariants. Our theorem above is consistent with this, since it is easy to verify in this case that for the line bundle $\lambda$ associated to the cover $\widetilde{Y} \rightarrow Y, \xi$ and $\xi \otimes \lambda$ are the only spin ${ }^{c}$ structures in $\mathcal{K}$ with non-trivial invariants: $S W(\xi)=S W(\xi \otimes \lambda) \neq 0$ (following [10]), so $S W(\widetilde{\xi})=0 \bmod 2$ from Theorem 4.2.

Finally we make an observation about complex line bundles whose Chern class is 2-torsion.

Proposition 4.6. Let $Y$ be a smooth. (not necessarily compact) manifold of any dimensions. For a complex line bundle $\lambda$ on $Y$, its Chern class $c_{1}(\lambda) \in H^{2}(Y, \mathbf{Z})$ is 2-torsion if and only if $\lambda$ is the complexification of a real line bundle on $Y$.

Proof. If $\lambda$ is the complexification of $\alpha, \lambda=\alpha \otimes \mathbf{C}$, then $\lambda^{\otimes 2}=\alpha^{\otimes 2} \otimes \mathbf{C}$. Of course $\alpha^{\otimes 2}$ is a trivial real line bundle; hence $\lambda^{\otimes 2}$ is trivial too and $2 c_{1}(\lambda)=c_{1}\left(\lambda^{\otimes 2}\right)=0$.

Conversely if $2 c_{1}(\lambda)=0$, then $\lambda$ is isomorphic to its dual and so to its conjugate: $\lambda \stackrel{\cong}{\longrightarrow}$. Composing with the anti-linear conjugation map $\bar{\lambda} \rightarrow \lambda$, one gets a map $u: \lambda \rightarrow \lambda$, which is an anti-linear isomorphism over each fiber. Since $u$ is an involution, $\lambda=\operatorname{Re} u \oplus \operatorname{Im} u$ as real bundles, where $\operatorname{Re} u=\operatorname{Fix} u$ and $\operatorname{Im} u=\{v \in \lambda \mid u(v)=-v\}$. Since $u$ is anti-linear, multiplying $\mathbf{i}$ gives an isomorphism $\operatorname{Re} u \rightarrow \operatorname{Im} u$. Hence $\lambda=\alpha \oplus \alpha$ as real bundles and consequently $\lambda=\alpha \otimes \mathbf{C}$, where $\alpha=\operatorname{Re} u$.

Note that even if the real line bundle $\alpha$ is nontrivial, $\alpha \oplus \alpha$ and hence the complexification $\lambda$ may still be trivial. Moreover from

$$
c_{1}(\lambda)=e(\alpha \oplus \alpha),
$$

one sees readily $c_{1}(\lambda)=w_{1}(\alpha)^{2} \bmod 2$.

\section{Topological preparations on branched covers.}

We set up our notations and collect together some elementary results that will be used for the next two sections. Let $\widetilde{X}, X$ be smooth 4-manifolds and 
$\Sigma \subset X$ be an embedded orientable connected surface. Consider a smooth map $p: \widetilde{X} \rightarrow X$ and put $\widetilde{\Sigma}=p^{-1}(\Sigma)$. (As a rule we shall always use the sign to indicate some pull-back object by $p$.) For our purpose, $p$ is an $m$-fold cover branched along $\Sigma$ if $p: \widetilde{X} \backslash \widetilde{\Sigma} \rightarrow X \backslash \Sigma$ is unramified $m$-fold cover, and $p$ has the form $z \mapsto z^{m}$ locally on the normal complex planes of $\widetilde{\Sigma}$ and $\Sigma$ in $\widetilde{X}$ and $X$. We take $m=2$, namely double branched cover, for the rest of the paper.

It is a familiar fact that a double branched cover exists if and only if $[\Sigma] \in H_{2}(X, \mathbf{Z})$ is divisible by 2 ; see for example Hirzebruch [7]. Granting the existence, the diffeomorphism type of $\widetilde{X}$ is then uniquely determined by $\Sigma$ and a class $a \in H_{2}(X, \mathbf{Z})$ such that $[\Sigma]=2 a$; so we will fix the class $a$ in order to fix the branched cover $\widetilde{X}$ and denote the induced line bundle by $\eta$ : $c_{1}(\eta)=P D(a)$.

Let $N$ be an open tubular neighborhood of $\Sigma \subset X ; N$ will also be viewed as the normal line bundle of $\Sigma \subset X$ when no confusion arises. The associated circle bundle is denoted by $\pi: S \rightarrow \Sigma$. Take $Y_{0}=X \backslash \operatorname{cl}(N)$ and $\widetilde{Y}_{0}=p^{-1}\left(Y_{0}\right)$ ("cl" denotes the closure). Then $p$ restricts to an unramified cover $p: \widetilde{Y}_{0} \rightarrow Y_{0}$.

Lemma 5.1. Let $n=[\Sigma]^{2}$ and $g$ be the genus of $\Sigma$ as before. Then we have

$$
H^{2}(S, \mathbf{Z}) \approx \mathbf{Z}^{2 g} \oplus \mathbf{Z} /(n \mathbf{Z}) .
$$

In particular when $n>0$, line bundles on $\Sigma$ all pull back to torsion bundles on $S$ via the projection $\pi: S \rightarrow \Sigma$.

Proof. Consider the Gysin sequence of the circle bundle $\pi: S \rightarrow \Sigma$ with coefficient group $\mathbf{Z}$ :

$$
\longrightarrow H^{0}(\Sigma) \stackrel{k}{\longrightarrow} H^{2}(\Sigma) \stackrel{\pi^{*}}{\longrightarrow} H^{2}(S) \longrightarrow H^{1}(\Sigma) \longrightarrow 0,
$$

where $k$ is the multiplication by the Euler characteristic of the circle bundle, namely $k(x)=n x$. Thus $H^{2}(S) \approx H^{1}(\Sigma) \oplus \operatorname{Im} \pi^{*}$ and $\operatorname{Im} \pi^{*} \approx H^{2}(\Sigma) / \operatorname{Im} k$, from which the result follows.

When $n>0$, the lemma simply reflects the fact that the normal bundle, having degree $n$, pulls back to the trivial bundle on $S$.

Recall for any smooth double un-ramified cover $\widetilde{Z} \rightarrow Z, \mathcal{K}_{Z}$ is defined to be the subgroup of $H^{2}(Z, \mathbf{Z})$ consisting of isomorphic line bundles on $Z$ that pull back to the trivial bundle on $\widetilde{Z}$. ( $\widetilde{Z}$ is suppressed in the notation 
of $\mathcal{K}_{Z}$ as the double cover will be self-evident in the following discussions.) Denote also $\mathcal{K}_{Z}^{*}=\mathcal{K}_{Z} \backslash\{0\}$. As remarked in Section 3, an application of Gysin sequence shows that $\mathcal{K}_{Z}^{*}$ is always a finite set of 2 -torsion elements.

Proposition 5.3. (1) The group $\mathcal{K}_{S} \subset H^{2}(S, \mathbf{Z})$ consists only of 0 and $[n / 2] \in \mathbf{Z} /(n \mathbf{Z}) \subset H^{2}(S, \mathbf{Z})$ if $n$ is even. Consequently any bundle in $\mathcal{K}_{Y_{0}}$, whose restriction on $S$ always belongs to $\mathcal{K}_{S}$, can be extended to a bundle on $X$.

(2) If $[\Sigma] \in H_{2}(X, \mathbf{Z})$ is not a torsion element of odd order, then $\mathcal{K}_{Y_{0}}^{*}$ is non-empty, in fact $\left.\eta\right|_{Y_{0}} \in \mathcal{K}_{Y_{0}}^{*}$.

(3) If $H_{1}(X, \mathbf{Z})$ contains no 2-torsion, then $\mathcal{K}_{Y_{0}}^{*}$ has at most one element.

Proof. (1) It is obvious, since $[n / 2] \in \mathbf{Z} /(n \mathbf{Z})$ is the only possible 2 -torsion in $H^{2}(S, \mathbf{Z})$ by Lemma 5.1 .

(2) We just need to show $\left.\eta\right|_{Y_{0}} \neq 0$. In the cohomology sequence with $\mathbf{Z}$ coefficients

$$
H^{2}\left(X, Y_{0}\right) \stackrel{i^{*}}{\longrightarrow} H^{2}(X) \stackrel{j^{*}}{\longrightarrow} H^{2}\left(Y_{0}\right)
$$

we have $H^{2}\left(X, Y_{0}\right) \cong H^{2}(\operatorname{cl}(N), S) \cong H^{0}(\Sigma)$ by excision and the Thom. isomorphism. Thus $\operatorname{Im} i^{*}$ is precisely the set of multiples of $P D[\Sigma]$. Of course $c_{1}\left(\eta \mid Y_{0}\right)=j^{*}(P D(a))$. If $c_{1}\left(\left.\eta\right|_{Y_{0}}\right)=0$, then $P D(a)=m P D[\Sigma]$ for some $m$, and hence $(2 m-1) P D[\Sigma]=0$ in view of $P D(a)=P D[\Sigma] / 2$. This contradicts the assumption.

(3) It suffices to verify that $H^{2}\left(Y_{0}\right)$ has at most one 2-torsion element. By universal coefficient theorem, it is enough to show $H_{1}\left(Y_{0}\right)$ has at most one 2-torsion. Consider the exact sequence of $\left(X, Y_{0}\right)$ :

$$
H_{2}(X) \stackrel{i_{*}}{\longrightarrow} H_{2}\left(X, Y_{0}\right) \stackrel{b}{\longrightarrow} H_{1}\left(Y_{0}\right) \stackrel{j_{*}}{\longrightarrow} H_{1}(X) .
$$

By excision and Thom isomorphism $H_{2}\left(X, Y_{0}\right) \cong H_{0}(\Sigma) \cong \mathbf{Z}$. Thus

$$
\operatorname{Im} b \cong H_{2}(X, Y) / \operatorname{Im} i_{*} \cong \mathbf{Z}_{m}
$$

for some $m$ and hence it can contain at most one 2-torsion. On the other hand, since $H_{1}(X)$ has no 2-torsion, any 2-torsion in $H_{1}\left(Y_{0}\right)$ must be in ker $j_{*}$ and so in $\operatorname{Im} b$.

Remark. Part (2) of the proposition remains to be true under a weaker: assumption $0 \neq[\Sigma] \in H_{2}(X, \mathbf{Z})$ by using an argument involving extension 
groups and real line bundles. The condition in the proposition is good enough for later applications, as we shall require $P D[\Sigma] \cdot c_{1}(L) \neq 0$ which forces $[\Sigma]$ to be non-torsion.

We introduce some natural definitions to be used throughout the section:

Definition 5.4. (1) Given any line bundle $L \rightarrow X$, the virtual dimension with respect to $L$ is defined to be

$$
d_{L}=\frac{1}{4}\left[c_{1}(L)^{2}-\left(2 e_{X}+3 s_{X}\right)\right]
$$

where $e_{X}$ and $s_{X}$ are respectively the Euler characteristic and signature of $X$.

(2) The adjunction term of $\Sigma$ with respect to $L$ is defined to be

$$
J_{L}(\Sigma)=\left|P D[\Sigma] \cdot c_{1}(L)\right|+[\Sigma] \cdot[\Sigma]+e_{\Sigma}
$$

Convention. The two cases with $P D[\Sigma] \cdot c_{1}(L) \leq 0, P D[\Sigma] \cdot c_{1}(L) \geq 0$ are "dual" for the following discussions, and can be déalt with similarly. From now on, we shall stick to the case $P D[\Sigma] \cdot c_{1}(L) \leq 0$, which is consistent with the associated $\operatorname{spin}^{c}$ structure of a complex surface, where $L$ is negative canonical bundle and $L$ intersects negatively with a complex curve.

Proposition 5.7. Let $e_{Y_{0}}$ and $s_{Y_{0}}$ be the Euler characteristic and signature of $Y_{0}$. Then we have

$$
2 e_{X}+3 s_{X}=2 e_{Y_{0}}+3 s_{Y_{0}}+4-4 g+3[\Sigma]^{2},
$$

where $g$ is the genus of $\Sigma$ and $[\Sigma]^{2}=[\Sigma] \cdot[\Sigma]$.

Proof. From the additivity of Euler characteristic we have

$$
e_{X}=e_{Y_{0}}+e_{N}-e_{\left(Y_{0} \cap N\right)}=e_{Y_{0}}+2-2 g .
$$

For signature we have also the Novikov additivity: $s_{X}=s_{Y_{0}}+s_{\operatorname{cl}(N)}$. Of course $s_{\operatorname{cl}(N)}=[\Sigma]^{2}$. Putting all these formulas together we show the proposition. 
Proposition 5.8. (1) Suppose $L \rightarrow X$ be a line bundle such that $c_{1}(L)$. $P D[\Sigma] \leq 0$, and let $\widehat{L}=\widetilde{L}-P D[\widetilde{\Sigma}] \rightarrow \widetilde{X}$, where $\widetilde{L}=p^{*} L$. Then we have

$$
d_{\widehat{L}}=2 d_{L}+\frac{1}{2} J_{L}(\Sigma)
$$

(2) The adjunction terms are simply identical: $J_{\widehat{L}}(\widetilde{\Sigma})=J_{L}(\Sigma)$.

Proof. (1) Keeping in mind $P D[\widetilde{\Sigma}]=p^{*}(P D[\Sigma] / 2)$, it is straightforward to check (5.9) by using the Euler characteristic and signature formulae for the branched double cover $\widetilde{X} \rightarrow X$ :

$$
e_{\widetilde{X}}=2 e_{X}-e_{\Sigma}, s_{\widetilde{X}}=2 s_{X}-[\widetilde{\Sigma}]^{2} .
$$

(2) It is straightforward to verify $J_{\widehat{L}}(\widetilde{\Sigma})=J_{L}(\Sigma)$.

Remark. Another useful way to express $\widehat{L}$ is $\widehat{L}=p^{*}\left(L \otimes \eta^{-1}\right)$.

A metric on $\Sigma$ induces a complex structure on $\Sigma$ and hence also on the total space $N$ of the normal bundle of $\Sigma$. Let $K_{N}, K_{\Sigma}$ denote the canonical line bundles of $N$ and $\Sigma$ (the bundles are of course independent of the metric up to isomorphism). The next proposition gives the significance of $J_{L}(\Sigma)=0$, which will be used in the gluing process.

Proposition 5.10. Given a line bundle $L \rightarrow X$, then $J_{L}(\Sigma)=0$ if and only if the restriction $\left.L\right|_{N}$ is isomorphic to $K_{N}^{-1}$.

Proof. One needs to show that $J_{L}(\Sigma)=0$ if and only if $\left.\left.L\right|_{\Sigma} \approx K_{N}^{-1}\right|_{\Sigma}$. If $J_{L}(\Sigma)=0$ then

$$
c_{1}\left(\left.L\right|_{\Sigma}\right)=c_{1}(L) \cdot P D[\Sigma]=e_{\Sigma}+[\Sigma]^{2}=-c_{1}\left(K_{\Sigma}\right)+c_{1}\left(\left.P D[\Sigma]\right|_{\Sigma}\right)
$$

implying $\left.L\right|_{\Sigma} \approx K_{\Sigma}^{-1} \otimes\left(\left.P D[\Sigma]\right|_{\Sigma}\right)$. On the other hand

$$
c_{1}\left(\left.K_{N}\right|_{\Sigma}\right)=-\left.c_{2}\left(T_{W}\right)\right|_{\Sigma}=-c_{2}\left(T_{\Sigma} \oplus N\right)=c_{1}\left(K_{\Sigma}\right)-c_{1}\left(\left.P D[\Sigma]\right|_{\Sigma}\right),
$$

and consequently one has $\left.\left.L\right|_{\Sigma} \approx K_{N}^{-1}\right|_{\Sigma}$. Reversing the process one gets the converse. 
Remark. The adjunction equality $J_{L}(\Sigma)=0$ is satisfied by any complex curve $\Sigma$ in a complex surface $X$ with $L$ to be the negative canonical bundle $K^{-1}$. The equality is also satisfied by the "opposite" of complex curves, a real part $X_{\mathbf{R}}$, namely an orientable connected component of the fixed point set of an anti-holomorphic involution on $X$. (In this situation, $K^{-1}$. $P D\left[X_{\mathbf{R}}\right]=0$ and $e_{X_{\mathbf{R}}}+\left[X_{\mathbf{R}}\right]^{2}=0$.) In general if $L$ is a $\mathrm{SW}$ basic class on a smooth 4-manifold $X$, then $J_{L}(\Sigma) \leq 0$ for any surface $\Sigma$ with $[\Sigma]^{2} \geq 0$ [15], [14].

Consider now a $\operatorname{spin}^{c}$ structure $\xi$ on $X$ with the determinant bundle $L \rightarrow X$. Because $p: \widetilde{X} \rightarrow X$ is a branched cover, $\xi$ does not directly pull back to a $\operatorname{spin}^{c}$ structure on $\widetilde{X}$. Nevertheless, $\widehat{L}=\widetilde{L} \otimes P D[\widetilde{\Sigma}]^{-1}$ is a characteristic line bundle on $\widetilde{X}$ from the relation of Stieffel-Whitney class, $w_{2}(\widetilde{X})=w_{2}(X)-P D[\widetilde{\Sigma}] \bmod 2$. Therefore there are $\operatorname{spin}^{c}$ structures on $\widetilde{X}$, parametrized by 2 -torsion elements in $H^{2}(\widetilde{X}, \mathbf{Z})$, whose determinant bundles are $\widehat{L}$. When $H^{2}(\widetilde{X}, \mathbf{Z})$ contains 2-torsion, there does not seem to be a way to define a preferred $\operatorname{spin}^{c}$ structure on $\widetilde{X}$ with determinant $\widehat{L}$, similar to that there is no canonical Riemann metric on $\widetilde{X}$ arising from a given metric on $X$.

Proposition 5.11. There exists a spin ${ }^{c}$ structure on $\widetilde{X}$ with determinant $\widehat{L}$, denoted by $\widehat{\xi}$, whose restriction to $\widetilde{Y}_{0}$ is isomorphic to the pull-back spin ${ }^{c}$ structure of $\left.\xi\right|_{Y_{0}}$.

Proof. Consider first the case $[\Sigma]^{2}=0$. So also $[\widetilde{\Sigma}]^{2}=0$, and by Lemma $5.1, H^{2}(\widetilde{S}, \mathbf{Z})$ has no 2 -torsion. Thus we can glue the pull-back $p^{*}\left(\xi \mid Y_{0}\right)$ with the $\operatorname{spin}^{c}$ structure on $\widetilde{N}=p^{-1}(N)$ defined by the line bundle $\left.\widetilde{L}\right|_{\widetilde{N}}$ along the neck $\widetilde{S} \times[-1,1]$, since the two spin ${ }^{c}$ structures, with the same determinant bundles, are isomorphic on the neck. Let $\breve{\xi}$ be such a glued $\operatorname{spin}^{c}$ structure on $\widetilde{X}$ (not unique). It follows from a standard cohomology argument that $\operatorname{det} \breve{\xi}=\widehat{L}+2 m P D[\tilde{\Sigma}]$ for some integer $m$. The spin ${ }^{c}$ structure $\widehat{\xi}=\breve{\xi} \otimes P D[\widetilde{\Sigma}]^{-m}$ is what is required in the proposition.

Consider the other case $[\Sigma]^{2} \neq 0$. Here Lemma 5.1 tells that there is a single 2-torsion $\beta$ in $H^{2}(\widetilde{S}, \mathbf{Z})$, which is in fact given as $\beta=\left(\frac{1}{2} \widetilde{\Sigma}^{2}\right) \mathbf{1}$, where $\mathbf{1} \in H^{2}(\widetilde{S}, \mathbf{Z})$ is the pull-back of the generator of $H^{2}(\widetilde{\Sigma}, \mathbf{Z})$. Gluing as above, we have a $\operatorname{spin}^{c}$ structure $\breve{\xi}$ on $\widetilde{X}$ whose determinant is either $\widehat{L}+2 m P D[\widetilde{\Sigma}]$ or $\widetilde{L}+2 m P D[\widetilde{\Sigma}]$ for some integer $m$, where the ambiguity occurs because of $\beta$. As $\widehat{L}$ is a characteristic bundle, it must be $\widehat{L}+2 m P D[\widetilde{\Sigma}]$ that is the determinant. Furthermore using $\widetilde{\Sigma}^{2} \neq 0$ and the determinant restricts to $\widehat{L}$ 
on $\widetilde{\Sigma}$, we find that $m$ must be zero, and hence $\breve{\xi}$ is already the required $\operatorname{spin}^{c}$ structure.

Remark. The proof also shows that when $[\Sigma]^{2} \neq 0$ there is a unique way to glue $\operatorname{spin}^{c}$ structures on $Y_{0}$ and $N$, and when $[\Sigma]^{2}=0$, the glued $\operatorname{spin}^{c}$ structures on $X$ are parametrized by $2 m P D[\Sigma]$ for integer $m$.

\section{SW invariants on branched covers: the $[\Sigma]^{2}=0$ case.}

Continue the set-up introduced in Section 5. Assume in addition that $[\Sigma]^{2}=$ 0 and the determinant of the $\operatorname{spin}^{c}$ structure $\xi$ on $X$ satisfies the adjunction equality $J_{L}(\Sigma)=0$, which means that $L$ restricts to a degree $2 g-2$ bundle on $\Sigma$. Moreover we require the genus $g>1$ so the preceding restriction of $L$ is non-trivial.

The goal in this section is to relate the SW invariants on $\widetilde{X}$ and $X$. Our idea is to first rewrite the invariants on $\widetilde{X}, X$ in terms of relative invariants on the cylindrical extensions of the complements of the branched locus. Because the cylindrical extensions are unramified covers, we then relate the relative invariants using the procedure of Section 3.

We are going to use the relative SW invariant formula given in MorganSzabo-Taubes [15]; the basic set-up is recalled here for reader's convenience. Introduce the cylindrical extension

$$
Y=\operatorname{cl}\left(Y_{0}\right) \cup_{S}(S \times[0, \infty))
$$

with the extended $\operatorname{spin}^{c}$ structure from $\left.\xi\right|_{Y_{0}}$ and product metric on the cylinder, and consider the perturbed SW equations:

$$
\not D_{A} \phi=0, F_{A}^{+}=q(\phi)+i \mu+i h .
$$

Here $\mu \in \Omega_{+}^{2}(Y)$ is supported in $Y_{0}$, and $h \in \Omega_{+}^{2}(Y)$ is supported in $S \times[1, \infty)$ and determined by a harmonic 1 -form on $\Sigma$. More precisely, on $S \times[1, \infty)$, $h$ is the pull-back of the 2 -form on $S=\Sigma \times S^{1}$ :

$$
h=*_{S} \alpha+d t \wedge \alpha,
$$

where $\alpha$ is a small but non-zero harmonic 1 -form on $\Sigma$. Let $(A, \phi)$ be a finite energy solution, namely the Chern-Simons functional $C\left(A_{t}, \phi_{t}\right)$ is bounded for all $t$, where $\left(A_{t}, \phi_{t}\right)$ is the restriction of $(A, \phi)$ to $S \times\{t\} \subset Y$. Then it is 
proved in [15] that as $t \rightarrow \infty,\left(A_{t}, \phi_{t}\right)$ converges exponentially to the unique SW solution on $S$; moreover the action

$$
c(A, \phi)=\frac{-1}{4 \pi^{2}} \int_{Y} F_{A} \wedge F_{A}
$$

is finite and takes discrete values as $(A, \phi)$ varies.

Let $M_{c}\left(\left.\xi\right|_{Y}\right)$ denote the SW moduli space of all solutions to (6.1) with finite energy and fixed action $c$. As their limits on $S$ are irreducible, all solutions in $M_{c}\left(\left.\xi\right|_{Y}\right)$ are also irreducible. According to [15], the introducing of $h$ ensures the compactness of $M_{c}\left(\left.\xi\right|_{Y}\right)$. With the additional generic perturbation $\mu$, the index calculations and Fredholm operators argument show that $M_{c}\left(\left.\xi\right|_{Y}\right)$, if non-empty, is a smooth compact manifold of dimension

$$
\frac{1}{4}\left(c-2 e_{Y}-3 s_{Y}\right)
$$

The usual procedure gives the relative $S W$ invariant $S W_{c}\left(\left.\xi\right|_{Y}\right)$, which depends only on the topology of $Y$, the $\operatorname{spin}^{c}$ structure $\left.\xi\right|_{Y}$, the action $c$ and the orientation for $H^{1}(Y) \oplus H_{+}^{2}(Y)$.

If $c$ is chosen to be $c_{1}(L)^{2}-2 e_{\Sigma}$, then Proposition 5.7 shows that $M_{c}\left(\left.\xi\right|_{Y}\right)$ has the same virtual dimension as the SW moduli space $M(\xi)$ of $\xi$ on $X$. Furthermore, after a generic perturbation, there is a unique SW solution among all $\operatorname{spin}^{c}$ structures on $N$, which can then be glued with the solutions in $M_{c}\left(\left.\xi\right|_{Y}\right)$ to get all solutions in $M(\xi)$. This gluing in turn yields the following identification of the corresponding invariants [18; Corollary 9.8].

Theorem 6.4 (Morgan-Szabo-Taubes). Let $\Sigma \subset X$ be an embedded surface of genus $g>1$ such that $[\Sigma]^{2}=0$, and $Y$ be the cylindrical extension of the complement of a tubular neighborhood of $\Sigma$. Suppose $\xi$ is a spin ${ }^{c}$ structure on $X$ such that $J_{L}(\Sigma)=0$ for its determinant bundle $L$. Then the relative $S W$ invariant of $\left.\xi\right|_{Y}$ with respect to the action $c=c_{1}(L)^{2}-2 e_{\Sigma}$ is equal to the $S W$ invariant on $X$ :

$$
S W_{c}\left(\left.\xi\right|_{Y}\right)=S W(\xi)
$$

For the $\operatorname{spin}^{c}$ structure $\widehat{\xi}$ on $\widetilde{X}$ constructed in Proposition 5.11, its determinant $\widehat{L}$ satisfies $J_{\widehat{L}}(\widetilde{\Sigma})=J_{L}(\Sigma)=0$ by Proposition 5.8. Thus we can apply the above result to the cylindrical extension $\tilde{Y}=p^{-1}(Y)$, with respect to $\left.\widehat{\xi}\right|_{\tilde{Y}}$. 
Corollary 6.6. Under the same conditions as the previous proposition, the relative $S W$ invariant of $\left.\widehat{\xi}\right|_{\widetilde{Y}}$ with respect to the action

$$
\tilde{c}=c_{1}(\widehat{L})^{2}-2 e_{\widetilde{\Sigma}}
$$

is given as $S W_{\tilde{c}}\left(\left.\widehat{\xi}\right|_{\tilde{Y}}\right)=S W(\widehat{\xi})$.

Remark. Using $J_{L}(\Sigma)=0$, one sees that $\tilde{c}=2 c$ where $c$ is as in Theorem 6.4 .

As recalled above, after perturbations, there is only one SW solution on the cylindrical extension of the neighborhood $N$ [15], and this is the reason why only one value of the action $c$ is picked up in the gluing and consequently in Proposition 6.4. For other values of $c$, the corresponding relative invariants provide additional topological invariants for $Y$, even though they are not necessarily related to any invariants on the closed manifold $X$ alone. A specific case that will be useful to us is in the following situation: Recall that $\eta \rightarrow X$ is a line bundle that is Poincare dual to $a \in H_{2}(X, \mathbf{Z})$, where $a$ is such that $2 a=[\Sigma]$ and determines the branched cover $p: \widetilde{X} \rightarrow X$. Consider the twisted $\operatorname{spin}^{c}$ structure $\xi \otimes \eta$ on $X$, whose determinant is $L^{\prime}=L \otimes P D[\Sigma]$. Take the moduli space of finite energy Seiberg-Witten solutions on $\left.\left.\xi\right|_{Y} \otimes \eta\right|_{Y}$ with the same action $c=c_{1}(L)^{2}-e_{\Sigma}$ as before and the associated relative invariant $S W_{c}\left(\left.\left.\xi\right|_{Y} \otimes \eta\right|_{Y}\right)$. Notice that the application of Proposition 6.4 to $\xi \otimes \eta$ would require a different action value $c_{1}\left(L^{\prime}\right)^{2}-e_{\Sigma}$, so the relative invariant $S W_{c}\left(\left.\left.\xi\right|_{Y} \otimes \eta\right|_{Y}\right)$ can not be pushed to equal the absolute invariant $S W(\xi \otimes \eta)$ on $X$. Instead $S W_{c}\left(\left.\left.\xi\right|_{Y} \otimes \eta\right|_{Y}\right)$ is an invariant of the triple $(X, \Sigma, a)$, and may be viewed as an invariant of the cover $\widetilde{X} \rightarrow X$.

From Proposition 5.3, $\left.\eta\right|_{Y} \in \mathcal{K}_{Y}^{*}$. The above discussion can also be carried out for other line bundles $\gamma \in \mathcal{K}_{Y}^{*}$. Indeed, since $\gamma$ restricts trivially to $S$ by Proposition 5.3, it can be extended trivially over the neighborhood of $\Sigma$ and yields a bundle $\bar{\gamma}$ on $X$ satisfying $P D[\Sigma] \cdot c_{1}(\bar{\gamma})=0$. Thus the relative invariant $S W_{c}\left(\left.\xi\right|_{Y} \otimes \gamma\right)$ is well-defined, as $J_{L+2 \gamma}(\Sigma)=J_{L}(\Sigma)=0$.

Definition 6.7. For any $\operatorname{spin}^{c}$ structure $\xi$ on $X$ with $J_{L}(\Sigma)=0$, define $k_{\xi}(X, \Sigma)$ as follows:

$$
k_{\xi}(X, \Sigma)=\sum_{[\gamma] \in \mathcal{K}_{Y}^{*}} S W_{c}\left(\left.\xi\right|_{Y} \otimes \gamma\right)
$$

where $c=c_{1}(L)^{2}-2 e_{\Sigma}$. 
Remark. The $k_{\xi}(X, \Sigma)$ is an invariant of the 2-dimensional knot $\Sigma \subset X$.

We now come to the main results in this section.

Theorem 6.8. Let $p: \widetilde{X} \rightarrow X$ be a double cover branched along a surface $\Sigma$ with genus bigger than $1,[\Sigma]^{2}=0$, and such that $b_{2}^{+}(X), b_{2}^{+}(\widetilde{X})>1$. Suppose that $\xi$ is a spin ${ }^{c}$ structure on $X$ whose determinant bundle $L$ is such that $c_{1}(L) \cdot P D[\Sigma] \leq 0$, and the virtual dimension and adjunction term both vanish: $d_{L}=J_{L}(\Sigma)=0$. Moreover let $\widehat{\xi}$ be a spin ${ }^{c}$ structure on $\widetilde{X}$ whose determinant bundle is $\widehat{L}=p^{*} L \otimes P D[\widetilde{\Sigma}]^{-1}$ and whose restriction to $\widetilde{Y}_{0}$ is the pull-back of $\left.\xi\right|_{Y_{0}}$. (The existence of $\widehat{\xi}$ is established in Proposition 5.11.) Then the following equality holds:

$$
S W(\widehat{\xi})=S W(\xi)+k_{\xi}(X, \Sigma) \quad \bmod 2 .
$$

Proof. For the cylindrical extension $Y$ of $Y_{0}$, obviously $\widetilde{Y}=p^{-1}(Y)$ is the cylindrical extension of $\widetilde{Y}_{0}$ and $\widetilde{Y} \rightarrow Y$ is an unramified double cover.

For the $\operatorname{spin}^{c}$ structures $\xi \otimes \bar{\gamma}$, parametrized by $\gamma \in \mathcal{K}_{Y}$, choose the same generic perturbation $\mu$ and small form $h \neq 0$ as in (6.1), so that the relative moduli spaces $M_{c}\left(\left.\xi\right|_{Y} \otimes \gamma\right)$ for a given $c$ are smooth manifolds of the correct dimension $\frac{1}{4}\left(c-2 e_{Y}-3 s_{Y}\right)$. Let $M_{\widetilde{c}}\left(\left.\widetilde{\xi}\right|_{\widetilde{Y}}\right)$ be the relative moduli space with the pull-back perturbations $p^{*} \mu, p^{*} h$, and the action $\widetilde{c}=2 c$. Since $h=*_{S} \alpha+d t \wedge \alpha$ from (6.2), it is easy to see $p^{*} h=*_{\tilde{S}} \alpha+d t \wedge \alpha$, and hence $M_{\widetilde{c}}\left(\left.\widetilde{\xi}\right|_{\tilde{Y}}\right)$ is a compact (but probably non-smooth) space by [15].

If $\tau$ denotes the involution on $\left.\widehat{\xi}\right|_{\widetilde{Y}}=\left.\widetilde{\xi}\right|_{\widetilde{Y}}$, obtained using the projection $p:\left.\left.\widetilde{\xi}\right|_{\widetilde{Y}} \rightarrow \xi\right|_{Y}$, then Theorem 3.8 gives a homeomorphism:

$$
M_{\tilde{c}}\left(\left.\widehat{\xi}\right|_{\tilde{Y}}\right) \approx M_{c}\left(\left.\xi\right|_{Y}\right) \coprod\left(\coprod_{\gamma \in \mathcal{K}_{Y}^{*}} M_{c}\left(\left.\xi\right|_{Y} \otimes \gamma\right)\right) .
$$

(Note that the irreducibility, required in Theorem 3.8, is not an issue here, since all relative moduli spaces contain only irreducible solutions by the exponential convergence to the irreducible solutions on $S$ and $\widetilde{S}$.)

Suppose now $c=c_{1}(L)^{2}-e_{\Sigma}$ and so $\widetilde{c}=2 c=c_{1}(\widehat{L})^{2}-2 e_{\widetilde{\Sigma}}$. Since $d_{L}=J_{L}(\Sigma)=0$, it follows from Proposition 5.8 that also

$$
d_{\widehat{L}}=J_{\widehat{L}}(\widetilde{\Sigma})=0 \text {. }
$$

Thus Theorem 6.4 and its corollary can be applied and yield

$$
S W(\widehat{\xi})=S W_{\widetilde{c}}\left(\left.\widehat{\xi}\right|_{\tilde{Y}}\right), S W(\xi)=S W_{c}\left(\left.\xi\right|_{Y}\right) .
$$


Moreover $M_{c}\left(\left.\xi\right|_{Y} \otimes \gamma\right), \gamma \in \mathcal{K}_{Y}$ all have dimension $\frac{1}{4}\left(c-2 e_{Y}-3 s_{Y}\right)=0$. Thus the relative invariants obey

$$
S W_{c}\left(\left.\xi\right|_{Y}\right)=\# M_{c}\left(\left.\xi\right|_{Y}\right), k_{\xi}(X, \Sigma)=\sum_{\gamma \in \mathcal{K}_{Y}^{*}} \# M_{c}\left(\left.\xi\right|_{Y} \otimes \gamma\right)
$$

As for $S W(\widehat{\xi})$, an application of Theorem 2.2 to $\left.\widehat{\xi}\right|_{\widetilde{Y}}=\left.\widetilde{\xi}\right|_{\widetilde{Y}}$ with the involution $\tau$ gives $S W_{\widetilde{c}}\left(\left.\widehat{\xi}\right|_{\tilde{Y}}\right)=\# M_{\widetilde{c}}\left(\left.\widehat{\xi}\right|_{\tilde{Y}}\right)^{\tau} \bmod 2$; thus also

$$
S W(\widehat{\xi})=\# M_{\tilde{c}}\left(\left.\widehat{\xi}\right|_{\tilde{Y}}\right)^{\tau} \bmod 2 .
$$

The combination of (6.10)-(6.12) together proves the formula (6.9).

We haven't found any example such that $k_{\xi}(X, \Sigma) \neq 0$ although we believe it should exist. The closest thing we come up, as pointed out by Selman Akbulut, is to take a connected sum of branched covers. First note that there is a double cover $S^{3} \rightarrow Q$ branched along the circle $\Gamma$, where $Q$ is a diffeomorphism copy of $Q$ (one can use the involution $(x, y, z, w) \mapsto$ $(x, y,-z,-w)$ on $\left.\mathbf{R}^{4}\right)$. Given any two double covers $\widetilde{X}_{i} \rightarrow X_{i}$ branched along $\Sigma_{i}$ with $i=1,2$, one can cut a small disk $D_{i}$ at a point on $\Sigma_{i}$ such that $\left(D_{i}, D_{i} \cap \Sigma\right)$ is diffeomorphic to $(Q, \Gamma)$. In view of the branched cover $S^{3} \rightarrow Q$, the connected sum operation can be performed and gives rise to a well-defined double cover

$$
\widetilde{X}_{1} \# \widetilde{X}_{2} \rightarrow X_{1} \# X_{2}
$$

branched along $\Sigma_{1} \# \Sigma_{2}$.

Now take $\left(X_{1}, \Sigma_{1}\right)$ to be a complex surface together with a smooth complex curve, and take $\left(X_{2}, \Sigma_{2}\right)$ to be $\left(S^{4}, T\right)$ as the quotient of $S^{2} \times S^{2}$ under the involution that is the diagonal complex conjugation on

$$
S^{2} \times S^{2}=\mathbf{C P}^{1} \times \mathbf{C P}^{1}
$$

( $T$ is the torus $S^{1} \times S^{1}$ consisting of the fixed points). By taking connected sum, we have a double cover $\widetilde{X}_{1} \#\left(S^{2} \times S^{2}\right)$ of $X_{1}$ branched along $\Sigma_{1} \# T$. If this branched cover satisfied the assumptions of Theorem 6.8 , we would have $k_{\xi}\left(X_{1}, \Sigma_{1} \# T\right) \neq 0$. However since the complex curve $\Sigma_{1}$ already satisfies the adjunction equality, the surface $\Sigma_{1} \# T$ does not and Theorem 6.8 does not apply.

We exclude the torus case $\Sigma=T$ in Theorem 6.8 , because the issue of reducible SW solutions on $S$ needs to be handled differently in the gluing 
formula. By using a forthcoming work of Mrowka, Morgan and Szabo, it may not be difficult to extend Theorem 6.8 to cover the torus case. Here we are content to point out an example (due to Akbulut again) which may be useful for this situation. Namely we take a connected sum of any 4manifold $X$ with the above branched cover $S^{2} \times S^{2} \rightarrow S^{4}$, where this time the connected sum is taken away from the branched torus $T$. The result is a double cover $\widetilde{X}=S^{2} \times S^{2} \# 2 X \rightarrow X=S^{4} \# X$ branched along $T$, with $[T]^{2}=0$ and $J_{L}(T)=0$ for any line bundle on $X$. (The analogue of this construction in the unramified case is the Kotschick-Morgan-Taubes example [10].)

\section{SW invariants on branched covers: the $[\Sigma]^{2}>0$ case.}

Assume the set-up as in Section 5, but this time we investigate the case $n=[\Sigma]^{2}>0$. It is worth pointing out that a distinguished role is played by the adjunction term $J_{L}(\Sigma)$. We shall make the key assumption that the adjunction equality holds: $J_{L}(\Sigma)=0$; this will be important in both the gluing of SW invariants and the requirement of the zero-dimensionality of the SW moduli space on $\widetilde{X}$. The equality $J_{L}(\Sigma)=0$ indicates that in essence we deal with the complex surface picture locally around the branched locus $\Sigma$.

We shall need a formula for a relative SW invariants which follows almost directly from the description of SW moduli space on the circle bundle $\pi$ : $S \rightarrow \Sigma$ by Mrowka-Ozsvath-Yu [18]. In order to put things in perspective, we need to explain briefly the set-up which they use.

Endow $S$ with the metric

$$
g_{S}=\theta+\pi^{*} g_{\Sigma}
$$

where $i \theta$ is a connection 1-form on the circle bundle $S \rightarrow \Sigma$ and $g_{\Sigma}$ is a metric of constant curvature. In order to define a variant of SW equations on $S$, the important observation made in [18] is to use the reducible connection ${ }^{\circ} \nabla=\left(d, \pi^{*} \nabla_{\Sigma}\right)$ on the tangent bundle

$$
T_{S}=\mathbf{R} \oplus \pi^{*} T_{\Sigma}
$$

induced by the Levi-Civita connection $\nabla_{\Sigma}$ of $g_{\Sigma}$, rather than the usual choice of Levi-Civita connection of $g_{S}$.

By using a base $\operatorname{spin}^{c}$ structure such as $W_{0}=\mathbf{C} \oplus \pi^{*} K_{\Sigma}^{-1}$, where $K_{\Sigma}$ is the canonical bundle on $\Sigma$, one can write any other $\operatorname{spin}^{c}$ structure $W$ on $S$ 
uniquely as $W=W_{0} \otimes E$ for a line $E$ on $S$. By tensoring with a canonically defined connection on $W_{0}$ [18; formula (24)], a Hermitian connection $A$ on $E$ induces a ${ }^{\circ} \nabla$-compatible connection on $W$ (called ${ }^{\circ} \nabla$-spinorial in [18]), which is coupled with ${ }^{\circ} \nabla$ to give the Dirac operator $\not_{A}$ as well as the SW equations on $W$ :

$$
\not D_{A} \psi=0, *_{S} \operatorname{Tr}\left(F_{A}\right)=\tau(\psi),
$$

where $\psi$ is a section of $W$ and $F_{A}$ is the curvature of the compatible connection on $W$.

From Lemma 5.1, $H^{2}(S) \approx \mathbf{Z}^{2 g} \oplus \mathbf{Z}_{n}$ and the subgroup $\mathbf{Z}_{n}$ corresponds precisely to the image of $H^{2}(\Sigma) \approx \mathbf{Z}$ via the pull-back map $\pi^{*}$. (All cohomology groups have $\mathbf{Z}$-coefficients unless otherwise stated.) Of course the induced projection $\pi^{*}: \mathbf{Z} \rightarrow \mathbf{Z}_{n}$ is not injective; as observed in [18; Proposition 5.1.3], one can define a lifting map by including the datum of connections on the corresponding line bundles: Let $e^{\prime} \in \mathbf{Z}$ and $A^{\prime}$ be a connection on the bundle $E^{\prime}$ over $\Sigma$ corresponding to $e^{\prime}$. Then the map $q\left(e^{\prime}, A^{\prime}\right):=\left(\pi^{*}\left(e^{\prime}\right), \pi^{*} A^{\prime}\right)$ is a one-one correspondence between the pairs $\left(e^{\prime}, A^{\prime}\right)$ and the pairs $(e, A)$, where $e \in \mathbf{Z}_{n}$ and $A$ is a connection on the corresponding bundle $E$ over $S$ that has trivial holonomy along fiber circles of $\pi: S \rightarrow \Sigma$.

We can now spell out the details of [18; Corollary 1.0.5] that applies suitably to each individual $\operatorname{spin}^{c}$ structure on $S$.

Theorem 7.3 (Mrowka-Ozsvath-Yu). Suppose the genus $g$ of $\Sigma$ is bigger than 1 and $0<n \leq 2 g-2$. Given a line bundle $E \rightarrow S$ and its spinc structure $W=W_{0} \otimes E$ on $S$, suppose the associated $S W$ moduli space $M_{S}(E)$ contains an irreducible solution. Then the following hold:

(1) The Chern class $c_{1}(E) \in \mathbf{Z}_{n} \subset H^{2}(S)$, and for every irreducible $(A, \psi) \in M_{S}(E)^{*}, A$ has trivial holonomy along fiber circles; so $\left(c_{1}(E), A\right)=q\left(e^{\prime}, A^{\prime}\right)$ for a unique pair $\left(e^{\prime}, A^{\prime}\right)$.

(2) Furthermore, if $c_{1}(E)$ is represented by $e \in \mathbf{Z}$ with $0 \leq e<n$, then $M_{S}(E)$ is identified with the union:

$$
T^{2 g} \coprod\left(\coprod_{i \in I} \operatorname{Sym}^{e+i n}(\Sigma)\right) \coprod\left(\coprod_{j \in J} \operatorname{Sym}^{2 g-2-(e+j n)}(\Sigma)\right),
$$

where $T^{2 g}$ is a $2 g$-torus, "Sym" means a symmetric product, $I=\{i \in$ $\left.\mathbf{Z}_{\geq 0} \mid e+i n<g-1\right\}$, and $J=\left\{j \in \mathbf{Z}_{\geq 0} \mid g-1<e+j n \leq 2 g-2\right\}$. 
Remark. The statement can be modified without the assumption $n \leq$ $2 g-2$. We impose the assumption here because in our case with $\Sigma \subset X$, the existence of a SW basic class on $X$ will imply $n \leq 2 g-2$ via the adjunction formula. (Originally a well-known theorem of Kronheimer-Mrowka [12] states that the inequality also follows from the non-triviality of the Donaldson invariants.) This also explains why we exclude the cases with the genus $g=0,1$, since $n>0$.

More precisely, the torus part in (7.4) corresponds to reducible solutions in $M_{S}(E)$. As for an irreducible pair $(A, \psi)$, let $\left(e^{\prime}, A^{\prime}\right)$ satisfy $\left(c_{1}(E), A\right)=$ $q\left(e^{\prime}, A^{\prime}\right)$, where $0 \leq e^{\prime} \leq 2 g-2$ and $e^{\prime} \neq g-1$. If $e^{\prime}<g-1$ then $(A, \psi)$ is the pull-back of a unique solution $\left(A^{\prime}, \alpha\right)$ to the equations:

$$
2 F_{A^{\prime}}-F_{K_{\Sigma}}=\mathbf{i}|\alpha|^{2} * 1_{\Sigma}, \partial_{A^{\prime}} \alpha=0,
$$

where $\alpha \neq 0$ is a section of $E^{\prime}$. Fixing $e^{\prime}$, the set of all solutions $\left(A^{\prime}, \alpha\right)$ is identified with $\operatorname{Sym}^{e^{\prime}}(\Sigma)$ by solving the Kazdan-Warner equation. If $e^{\prime}>$ $g-1$ then equations (7.5) are replaced by their dual:

$$
2 F_{A^{\prime}}-F_{K_{\Sigma}}=-\mathbf{i}|\beta|^{2} * 1_{\Sigma}, \bar{\partial}_{A^{\prime}}^{*} \beta=0,
$$

where $0 \neq \beta \in \Gamma\left(K_{\Sigma}^{-1} \otimes E^{\prime}\right)$; the solutions $\left(A^{\prime}, \beta\right)$ are parametrized by $\operatorname{Sym}^{2 g-2-e^{\prime}}(\Sigma)$.

For the purpose of the gluing, we also need to understand the SW solutions on the cylindrical tubular neighborhood

$$
N=N_{0} \cup_{\partial N_{0}=S}[-1, \infty) \times S,
$$

endowed with the metric $g_{\text {cyl }}=d t^{2}+g_{S}$. The natural complex structure on $N$ gives a canonical $\operatorname{spin}^{c}$ structure

$$
W_{0}^{+}=\mathbf{C} \oplus K_{N}^{-1}, W_{0}^{-}=\wedge_{N}^{0,1} .
$$

Viewing $S=\partial N_{0}$, it is easy to see $\left.W_{0}^{+}\right|_{\partial N_{0}}=\mathbf{C} \oplus p^{*} K_{\Sigma}^{-1}=W_{0}$; moreover, using the inward normal vector of $\partial N_{0}$ in $N_{0}$, the $\operatorname{spin}^{c}$ structure on $N_{0}$ induces one on the boundary $\partial N_{0}$, which is precisely the $\operatorname{spin}^{c}$ structure $W_{0}$ on $S$.

It turns out in order to make use of the complex structure on $N$, it is more convenient to use a compatible Kähler metric $g$ rather than the cylindrical metric $g_{\text {cyl }}$. According to [13], $g$ can be obtained by extending it from the cylinder $[0, \infty) \times S$ where it is conformal to $g_{\text {cyl }}, g=e^{-n t} g_{\text {cyl }}$, the last formula being observed by G.-Y. Guo for the first time. The point is that 
there is a well-defined $\operatorname{Spin}^{c}(4)$ bundle on $N$ determined by the conformal class of $g_{\text {cyl }}$, and so the Dirac operators of $g, g_{\text {cyl }}$ can be compared on the same bundle, cf. Hitchin [8]. Thus, as proved by [17], there is a one-to-one correspondence between the SW moduli spaces on $N$ that use the metrics $g$ and $g_{\text {cyl }}$. Of course using $g$ and $W_{0}^{ \pm}$, the SW moduli space for the $\operatorname{spin}^{c}$ structure $W_{0}^{ \pm} \otimes E$ is simply given by the set of solutions $(A ; \alpha, \beta)$ of

$$
\begin{aligned}
2 \Lambda F_{A}-\Lambda F_{K_{N}} & =\mathbf{i}\left(|\alpha|^{2}-|\beta|^{2}\right), \\
2 F_{A}^{0.2}-F_{K_{N}}^{0,2} & =\alpha^{*} \otimes \beta, \\
\bar{\partial}_{A} \alpha+\bar{\partial}_{A}^{*} \beta & =0
\end{aligned}
$$

where $A$ is a connection on $E$ and $\psi=(\alpha, \beta) \in \Gamma\left(E \oplus E \otimes K_{N}^{-1}\right)$.

Proposition 7.8. For any $(A, \psi) \in M_{S}(E)^{*}$ with $\left(c_{1}(E), A\right)=q\left(e^{\prime}, A^{\prime}\right)$, there is an $S W$ solution for the spin ${ }^{c}$ structure $W_{0}^{ \pm} \otimes \pi^{*} E^{\prime}$, which converges exponentially to $(A, \psi)$ on the cylinder $[0, \infty) \times S$.

Proof. We consider the case $e<g-1$; the other case $g-1<e$ is entirely similar. Here $(A, \psi)$ is the pull-back, from $\Sigma$ to $S$, of a vortex solution $\left(A^{\prime}, \alpha\right)$ to $(7.5)$ and defined on $E^{\prime} \rightarrow \Sigma$. The projection $\pi: N \rightarrow \Sigma$ also pulls back the vortex to a pair $\left(\pi^{*} A^{\prime}, \pi^{*} \alpha\right)$ defined on $W_{0}^{+} \otimes \pi^{*} E^{\prime}$. Then we need to find a real valued function $u$ on $N$ such that $e^{u}\left(\pi^{*} A^{\prime}, \pi^{*} \alpha\right)$ satisfies equations (7.7) above, which are equivalent to the single Kazdan-Warner type equation:

$$
\Delta u+\frac{\left|\pi^{*} \alpha\right|^{2}}{2} e^{2 u}+\mathbf{i} \wedge \pi^{*} F_{A^{\prime}}-\frac{\mathbf{i}}{2} \wedge F_{K_{N}}=0 .
$$

The last equation can be solved using the usual upper-lower solution method, and the key is to construct upper and lower solutions. The exponential. convergence on the cylinder follows from that $u$ converges to zero on the cylinder. For details, compare with [18; section 8$]$.

We proceed to examine the relative invariant problem in the context that we will need. To that end we shall use Theorem 7.3 and Proposition. 7.8 only in a rather special situation. For the rest of the paper, we continue using the notations as introduced in Section 5, and assume again the $\operatorname{spin}^{c}$ structure $\xi$ on $X$ is such that

$$
J_{L}(\Sigma)=-c_{1}(L) \cdot P D[\Sigma]+2-2 g+n=0 .
$$


Then by Proposition 5.10, the restriction $\xi_{N}$ is the same as the canonical $\operatorname{spin}^{c}$ structure $W_{0}^{ \pm}$on $N$ introduced above; thus the further restriction $\xi_{S}$ is just $W_{0}$ corresponding to $e=0 \in \mathbf{Z}_{n}$. Let $u_{0} \in M_{S}(E)$ be the unique irreducible solution in $\operatorname{Sym}^{0}(\Sigma)$ in the decomposition 7.4, namely $u_{0}$ is the pull-back of $(0,1)$ where 0 is the trivial connection and 1 is the constant section, both on the trivial bundle $E^{\prime}=\underline{\mathbf{C}} \rightarrow \Sigma$. Moreover we assume the virtual dimension $d_{L}(\Sigma)=0$. Put a cylindrical metric $g_{Y} d t^{2}+\theta^{2}+\pi^{*} g_{\Sigma}$ on $Y=Y_{0} \cup[-1, \infty) \times S$.

Definition 7.10. The relative invariant $S W\left(\left.\xi\right|_{Y}\right)$ of $\left.\xi\right|_{Y}$ is defined to be the sum of the finite energy solutions $(A, \phi)$, counted with suitable signs, to the perturbed SW equations of $\left.\xi\right|_{Y}$, such that

(1) the action $c(A, \phi)=\frac{-1}{4 \pi^{2}} \int_{Y} F_{A} \wedge F_{A}$ equals $c_{1}(L)^{2}-4+4 g+3 n^{2}$, and

(2) after gauge transformations, $(A, \phi)$ converges exponentially to $u_{0}$ on the cylinder of $Y$.

Strictly speaking, to use the SW equations (7.2) on $S$, we need to fix a connection $\nabla^{\prime}$ on the tangent bundle $T_{Y}$ that restricts to the reducible connection ${ }^{\circ} \nabla$ on the cylindrical part of $Y$. Then by using a $\nabla^{\prime}$-compatible connection on on $W_{0}^{+}$, other $\nabla^{\prime}$-compatible connections are in one-to-one correspondence with connections on the trivial C-bundle and the SW equations on $Y$ can then be defined in the usual way.

In the definition above, the moduli space of the finite energy solutions has dimension zero, following the usual index calculations. The compactness of the moduli space follows from the existence of a uniform bound on spinors as well as the fact that there is only one flow, namely the constant flow, on $\mathbf{R} \times S$ which connects $u_{0}$ and $u_{0}$ itself, see [18]; compare with the $[\Sigma]^{2}=0$ case in [15].

Remark. Our discussion of relative invariants here is undoubtedly very concentrated; for a more complete account see a recent paper by Ozsvath and Szabo [19].

We are in the position to state a relative invariant theorem; the analogue for Donaldson invariants has been shown by Kronheimer-Mrowka [12; Proposition 5.8].

Theorem 7.11. Let $\Sigma \subset X$ be an embedded surface with

$$
0<n=[\Sigma]^{2} \leq 2 g-2 .
$$


Suppose $2 g-2$ is not divisible by $n$. If $\xi$ is a spin ${ }^{c}$ structure on $X$ such that $d_{L}=J_{L}(\Sigma)=0$ for its determinant bundle $L$, then we have

$$
S W\left(\left.\xi\right|_{Y}\right)=S W(\xi) .
$$

Proof. As usual consider $X_{r}=Y_{0} \cup([-r, r] \times S) \cup N$ with a neck of length $2 r$ inserted in the middle. Extend the the product metric $d t^{2}+g_{S}$ from the neck to a metric on $X_{r}$ and fix a connection $\nabla^{\prime}$ on $T_{X}$, obtained by gluing properly the pull-back connection of ${ }^{\circ} \nabla$ on the neck with the Levi-Civita connections on the interiors of $Y_{0}$ and $N$. Then using [18; Lemma 5.2.1], the difference of the Dirac operators on $W^{+}$associated with $\nabla^{\prime}$ and $\nabla_{g}$ is a compact operator, and hence the SW invariants defined by using $\nabla^{\prime}$ and $\nabla_{g}$ are the same. We shall keep $\nabla^{\prime}$ in our discussions from now on.

The following line of arguments is similar to the instanton case of [12]. Consider the relative moduli space $M\left(\left.\xi\right|_{Y}\right)$ of SW solutions on the cylindrical extension $Y=Y_{0} \cup([0, \infty) \times S)$ that satisfy the conditions in Definition 7.10. Note that the solution $u_{0}$ on $S$ extends uniquely to a $S W$ solution $u_{0}^{\prime}$ on the cylindrical extension $\bar{N} \cup([0, \infty) \times S)$, since only the constant flow connects $u_{0}$ with $u_{0}$ by [18]. According to the general argument [16], for any $(A, \phi) \in M\left(\left.\xi\right|_{Y}\right)$, we can glue the restriction $\left.(A, \phi)\right|_{Y_{0} \cup[0, r] \times S}$ with $\left.u_{0}^{\prime}\right|_{\bar{N}} \cup([0, r] \times S)$ and deform it into a SW solution. In other words, we have the injective map

$$
\Upsilon_{r}: M\left(\left.\xi\right|_{Y}\right) \rightarrow M\left(\xi ; X_{r}\right)
$$

To complete the proof, we just need to show that $\Upsilon_{r}$ is surjective for sufficiently large $r$. Suppose the contrary that there is a sequence $r_{i} \rightarrow \infty$ together with solutions $v_{i} \in M\left(\xi ; X_{r_{i}}\right)$ which do not lie in the image of $\Upsilon_{i}$. We will obtain a contradiction as follows.

Imitating the compactness argument of [14], one shows that $v_{i}$ has a convergent subsequence, $v_{i} \rightarrow\left(v^{\prime}, w_{1}, \cdots, w_{k}, v^{\prime \prime}\right)$, where $v^{\prime}, v^{\prime \prime}$ are finite energy SW solutions on $Y$ and $N$ and $w_{i}$ are finite nonzero energy SW solutions on the tube $S \times \mathbf{R}$, respectively. Furthermore, the boundary value of $v^{\prime}$ matches the $-\infty$ boundary value of $w_{1}$, the $+\infty$-boundary value of $w_{1}$ matches the $-\infty$ boundary value of $w_{2}$ and so on. By Theorem 9.2.5 of [18], $v^{\prime}, w_{i}, v^{\prime \prime}$ are smooth points of the corresponding moduli spaces. Therefore, each moduli space has a nonnegative virtual dimension. By the additivity of the index, the virtual dimension of $v_{i}>0$ unless no $w_{i}$ appears in the limit. We can also view this as follow. If some $w_{i}$ appears in the limit, we construct an open subset of the moduli space by the gluing argument. Then, we notice that there is a gluing parameter $\mathbf{R}^{+}$corresponding to $w_{i}$. This contradicts 
the zero dimensionality of the moduli space. From the assumption, $g-1$ is not divisible by $n$, so Theorem 7.3 says that $M_{S}(E)$, with $E$ trivial here, does not contain the torus component that consists of pull-back reducible solutions from $\Sigma$; thus all solutions in $M_{S}(E)$ are non-degenerate by [18; Propositions 5.8.4, 5.9.4]. Hence, along the general line of [16], both $v^{\prime}$ and $v^{\prime \prime}$ converge exponentially to some elements $u^{\prime}, u^{\prime \prime} \in M_{S}(E)$ on the cylinders of $Y$ and $N$. We intend to show that $u^{\prime}=u^{\prime \prime}=u_{0}$ by counting dimensions.

In fact, since $2 g-2$ is not divisible by $n$, from (7.4) the moduli space $M_{S}(E)$ has only one zero-dimensional component $\left\{u_{0}\right\}$. So if $u^{\prime} \neq u_{0}$ then $u^{\prime}$ must belong to $T^{2 g}$ or $\operatorname{Sym}^{r}(\Sigma)$ from some $r>0$ as in (7.4). Let $c^{\prime}, c^{\prime \prime}$ be the actions of $v^{\prime}, v^{\prime \prime}$. Then $c_{1}(L)=c^{\prime}+c^{\prime \prime}$ from the convergence of $v_{i}$. Let $M\left(\left.\xi\right|_{Y} ; B\right)$ be the moduli space of SW solutions on $Y$ which converge to elements in $B$, where $B$ is either the torus $T^{2 g}$ or $\operatorname{Sym}^{r}(\Sigma)$; let $M\left(\left.\xi\right|_{N} ; B\right)$ be similarly defined. The dimension formula from the gluing theorem [16] is

$$
\operatorname{dim} M(\xi)=\operatorname{dim} M\left(\left.\xi\right|_{Y} ; B\right)+\operatorname{dim} M\left(\left.\xi\right|_{Y} ; B\right)-m,
$$

where $m>0$ is either $2 g-1$ or $2 r$, depending on $B$ is $T^{2 g}$ or $\operatorname{Sym}^{r}(\Sigma)$. On the other hand,

$$
\begin{aligned}
4\left[\operatorname{dim} M\left(\left.\xi\right|_{Y} ; B\right)+\operatorname{dim} M\left(\left.\xi\right|_{N} ; B\right)\right] & =c^{\prime}+c^{\prime \prime}-\left(2 e_{Y}+3 s_{Y}+2 e_{N}+3 s_{N}\right) \\
& =c_{1}(L)-\left(2 e_{X}+3 s_{X}\right) \\
& =4 \operatorname{dim} M(\xi)
\end{aligned}
$$

using Proposition 5.7 for the middle step, which forces $m=0$ and $u^{\prime}=u^{\prime \prime}=$ $u_{0}$.

Now that both $v^{\prime}$ and $v^{\prime \prime}$ converge to $u_{0}$ on the cylinders, the gluing argument of [16] can be applied once more to show that $v_{i}$ should lie in the image of $\Upsilon_{i}$ for large $i$. So the desired contradiction is obtained.

In the theorem we assumed $2 g-2$ is not divisible by $n$, in particular $n \neq$ $2 g-2$, in order to avoid the torus $T^{2 g}$ of degenerate reducible solutions on $S$ which are pull-backs from the bundle $E^{\prime} \rightarrow \Sigma$ with $e^{\prime}=g-1$. The inequality $n \neq 2 g-2$ implies that $L$ restricts non-trivially on $\Sigma$ from $J_{L}(\Sigma)=0$.

The following is the main result in the section; all required conditions are stated in full for completeness.

Theorem 7.14. Let $p: \widetilde{X} \rightarrow X$ be a double cover branched along a connected orientable surface $\Sigma$, with $n=[\Sigma]^{2}>0, b_{2}^{+}(X)>1, b_{2}^{+}(\widetilde{X})>1$, and $H_{1}(X, \mathbf{Z})$ containing no 2-torsion. Suppose $n<2 g-2$ and $4 g-4$ is 
not divisible by $n$. Suppose also that $\xi$ is a spin ${ }^{c}$ structure on $X$ such that $d_{L}=J_{L}(\Sigma)=0$ for its determinant bundle $L$. If $\widehat{\xi}$ is a spin ${ }^{c}$ structure defined in Proposition 5.11, then we have

$$
S W(\widehat{\xi})=S W(\xi) \bmod 2 .
$$

Proof. Fix connections $\nabla^{\prime}, \widehat{\nabla}^{\prime}$ on the tangent bundles $T_{X}, T_{\widetilde{X}}$ with the property that $\nabla^{\prime}$ and $\hat{\nabla}^{\prime}$ restrict to the reducible connections ${ }^{\circ} \nabla$ on $N$ and $\widetilde{N}$, and $\widehat{\nabla}^{\prime}$ is the pull-back of $\nabla^{\prime}$ on $\widetilde{Y}$. By tenoring with a suitable connection on $W^{+}$that is compatible with the decomposition $\left.W^{+}\right|_{N}=\mathbf{C} \oplus K_{N}$, the SW equations on $\xi=\xi \otimes E$ are defined for pairs $(A, \phi)$ where $A$ is a connection on the trivial line bundle $E$ and $\phi \in \Gamma\left(W^{+}\right)$; similarly for $\widehat{\xi}=\widehat{\xi} \otimes \widehat{E}$ with $\widehat{E}$ trivial.

With a generic perturbation in the SW equations for $\xi$, we have from Theorem 7.11 that $S W(\xi)=\# M\left(\left.\xi\right|_{Y}, u_{0}\right)$, where $M\left(\left.\xi\right|_{Y}, u_{0}\right)$ is the space of SW solutions on $Y$ satisfying the conditions of Definition 7.10. Define $M\left(\left.\widehat{\xi}\right|_{\widetilde{Y}}, \widetilde{u}_{0}\right)$ similarly, where by our choice, $\left.\widehat{\xi}\right|_{\widetilde{Y}}$ is the pull-back $\left.\widetilde{\xi}\right|_{\widetilde{Y}}$ of $\left.\xi\right|_{Y}$ via the projection $p$. We will switch to the notation $\left.\widetilde{\xi}\right|_{\widetilde{Y}}$ for the sake of the argument.

It is clear that $M\left(\left.\xi\right|_{Y}, u_{0}\right)$ is contained in $M\left(\left.\widetilde{\xi}\right|_{\widetilde{Y}}, \widetilde{u}_{0}\right)$ through the pullback map $p^{*}$. (The solutions $u_{0}, \widetilde{u}_{0}$, defined on $S$ and $\widetilde{S}$, are the pull-backs of the same solution $(0,1)$ on $\Sigma=\widetilde{\Sigma}$.)

More precisely applying Theorem 3.8 we have the decomposition into equivariant moduli spaces:

$$
M\left(\left.\widetilde{\xi}\right|_{\widetilde{Y}}, \widetilde{u}_{0}\right)^{\tau}=M\left(\left.\widetilde{\xi}\right|_{\widetilde{Y}}, \widetilde{u}_{0}\right)_{\tau} \coprod\left(\coprod_{k \in K_{\tilde{Y}}^{*}} M\left(\left.\widetilde{\xi}\right|_{\widetilde{Y}}, \widetilde{u}_{0}\right)_{k \tau}\right),
$$

where $\tau$ is the natural lifting on $\widetilde{\xi}_{\left.\right|_{\tilde{Y}}}$ and $K_{\widetilde{Y}}^{*}$ is the set of non-identity maps in $K_{\widetilde{Y}}$. (Again the irreducibility of the solutions is insured by that of $\widetilde{u}_{0}$.) From Proposition $5.3(2)$ and (3), $K_{\widetilde{Y}}^{*}$ contains precisely one map $k_{\eta}$ corresponding to the only line bundle $\left.\eta\right|_{Y}$ in $\mathcal{K}_{Y}^{*}$. Now we claim the component $M\left(\left.\widetilde{\xi}\right|_{\tilde{Y}}, \widetilde{u}_{0}\right)_{k_{\eta} \tau}$ is actually empty, and hence

$$
\coprod_{k \in K_{\tilde{Y}}^{*}} M\left(\left.\widetilde{\xi}\right|_{\tilde{Y}}, \widetilde{u}_{0}\right)_{k \tau}=\emptyset .
$$

In fact, if there is an element $[A, \phi] \in M\left(\left.\widetilde{\xi}\right|_{\widetilde{Y}}, \widetilde{u}_{0}\right)_{k_{\eta} \tau}$, then by looking at its limit we have $\tau^{*}\left(g \widetilde{u}_{0}\right)=k_{\eta} g \widetilde{u}_{0}$ for some gauge transformation $g$ on $\widetilde{\xi} \widetilde{S}_{\widetilde{S}}$. On 
the other hand, since $\widetilde{u}_{0}$ is the pull-back of $(0,1)$ via the fibration $\pi: \widetilde{\Sigma} \rightarrow \widetilde{S}$, obviously $\tau^{*} \widetilde{u}_{0}=\widetilde{u}_{0}$. Thus $\left.\left[\widetilde{u}_{0}\right] \in M(\widetilde{\xi})_{\widetilde{S}}\right)_{\tau}^{*} \cap M\left(\left.\widetilde{\xi}\right|_{\widetilde{S}}\right)_{k_{\eta} \tau}^{*}$ for the SW moduli space $M^{*}(\widetilde{\xi} \mid \widetilde{S})$ on $\widetilde{S}$ with the induced action $\tau$. This is however impossible: From Proposition $5.3(1),\left.\eta\right|_{\widetilde{S}}=[n / 4] \in \mathbf{Z}_{n / 2} \subset H^{2}(\widetilde{S}, \mathbf{Z})$ is non-zero, hence Theorem 3.8 applied to the moduli space e $M\left(\left.\widetilde{\xi}\right|_{\widetilde{S}}\right)^{*}$ yields

$$
M\left(\left.\widetilde{\xi}\right|_{\widetilde{S}}\right)_{\tau}^{*} \cap M\left(\left.\widetilde{\xi}\right|_{\widetilde{S}}\right)_{k_{\eta} \tau}^{*}=\emptyset
$$

(Theorem 3.8 is applicable here from the remark after that theorem.)

According to Theorem $3.8, M\left(\left.\xi\right|_{Y}, u_{0}\right)$ is homeomorphic to $M\left(\left.\widetilde{\xi}\right|_{\widetilde{Y}}, \widetilde{u}_{0}\right)_{\tau}$ via $p^{*}$; thus $M\left(\left.\widetilde{\xi}\right|_{\widetilde{Y}}, \widetilde{u}_{0}\right)^{\tau}$ is homeomorphic to $M\left(\left.\xi\right|_{Y}, u_{0}\right)$ from (7.16)-(7.17), and consequently $S W(\xi)=\# M\left(\left.\widetilde{\xi}\right|_{\tilde{Y}}, \widetilde{u}_{0}\right)^{\tau}$. On the other hand, by Theorem 2.2, \#M( $\left(\left.\widetilde{\xi}\right|_{\tilde{Y}}, \widetilde{u}_{0}\right)^{\tau}=S W\left(\left.\widetilde{\xi}\right|_{\widetilde{Y}}\right) \bmod 2$. Moreover using Proposition 5.8, we see that the conditions of Theorem 7.11 are satisfied by the $\operatorname{spin}^{c}$ structure $\widehat{\xi}$ and so $S W(\widehat{\xi})$ is equal to the relative invariant $S W\left(\left.\widehat{\xi}\right|_{\widetilde{Y}}\right)$. Putting all things together, we have $S W(\xi)=S W(\widehat{\xi}) \bmod 2$.

Remark. (1) If we drop the assumption that $H_{1}(X)$ contains no 2torsion, then $\mathcal{K}_{Y}^{*}$ may contain a non-trivial bundle $\gamma$ which restricts trivially on $S$. Namely the disjoint moduli spaces

$$
M\left(\left.\widetilde{\xi}\right|_{Y}, \widetilde{u}_{0}\right)_{\tau}, \quad M\left(\left.\widetilde{\xi}\right|_{\widetilde{Y}}, \widetilde{u}_{0}\right)_{k_{\gamma} \tau}
$$

may become intersected on their limits on $S$. Thus the non-emptiness of $M\left(\left.\widetilde{\xi}\right|_{\widetilde{Y}}, \widetilde{u}_{0}\right)_{k_{\gamma} \tau}$ can not be ruled out like $M\left(\left.\widetilde{\xi}\right|_{\widetilde{Y}}, \widetilde{u}_{0}\right)_{k_{\eta} \tau}$. It is not clear how to define the relative invariant from

$$
M\left(\left.\widetilde{\xi}\right|_{\tilde{Y}}, \widetilde{u}_{0}\right)_{k_{\gamma} \tau} \approx M\left(\left.\xi\right|_{Y} \otimes \gamma, u_{0}\right),
$$

since the adjunction equality is not valid for the $\operatorname{spin}^{c}$ structure $\xi \otimes \gamma$ and Definition 7.10 can not be used.

(2) Theorem 7.14 would be consistent with Theorem 4.2 if $\Sigma$ were allowed to be empty in Theorem 7.14: When $H_{1}(X, \mathbf{Z})$, or equivalently when $H^{2}(X, \mathbf{Z})$ has no 2-torsion, $\mathcal{K}_{X}=\{0\}$ and so Theorem 4.2 says $S W(\widetilde{\xi})=S W(\xi) \bmod 2$, coincident with (7.15). Putting differently, (4.3) suggests that (7.15) would not be true if $H_{1}(X, \mathbf{Z})$ has a 2 -torsion.

(3) Note that even if assuming $H_{1}(X)$ does not have 2-torsion for the case $[\Sigma]^{2}=0$ in Theorem 6.8 , we still can not drop the relative invariant 
term $k_{\xi}(X, \Sigma)$. This is just because $\left.\eta\right|_{Y}$ restricts trivially on $S$ unlike the case $[\Sigma]^{2}>0$. Such a difference between Theorems 7.14 and 6.8 is already hinted by the following: in the $[\Sigma]^{2}>0$ case, the adjunction inequality is violated for the $\operatorname{spin}^{c}$ structure $\xi \otimes \eta: J_{L+2 \eta}(\Sigma)=J_{L}(\Sigma)+$ $[\Sigma]^{2}=[\Sigma]^{2}>0$, whereas for the $[\Sigma]^{2}=0$ case the adjunction term $J_{L+2 \eta}(\Sigma)$ remains to be zero.

The next corollary generalizes the familiar formula from holomorphic covers of complex surfaces to smooth covers of complex surfaces; in spirit it is similar to that the canonical bundle of a minimal complex surface is invariant under smooth diffeomorphisms.

Corollary 7.18. Suppose that $X$ is a complex surface of general type with $b_{2}^{+}(X)>1$, the canonical bundle $K$, and without 2-torsion in $H_{1}(X, \mathbf{Z})$. If $p: \widetilde{X} \rightarrow X$ is a smooth double cover branched along an orientable connected surface $\Sigma$ and $\Sigma$ is such that $J_{K}(\Sigma)=0,0 \lesssim[\Sigma]^{2}<2 g-2$ and $4 g-4$ is not divisible by $[\Sigma]^{2}$, then $c_{1}\left(p^{*} K\right)+P D[\widetilde{\Sigma}]$ is a $S W$ basic class of $\tilde{X}$. If moreover $\widetilde{X}$ is also a complex surface of general type and both $X, \widetilde{X}$ are minimal, then $c_{1}(\widetilde{K})= \pm\left(c_{1}(K)+P D[\widetilde{\Sigma}]\right)$, where $\widetilde{K}$ is the canonical bundle of $\widetilde{X}$.

Proof. Let $\xi$ be the canonical $\operatorname{spin}^{c}$ structure on $X$; its determinant is $K^{-1}$ of course. By the familiar computation [26], $S W(\xi)=1$; hence $S W(\widehat{\xi}) \neq 0$ by Theorem 7.14, and so $S W\left(\widehat{\xi}^{*}\right) \neq 0$ for the dual $\widehat{\xi}^{*}$. Thus the determinant of $\widehat{\xi}^{*}$, namely $c_{1}\left(p^{*} K\right)+P D[\tilde{\Sigma}]$, is an SW basic class on $\widetilde{X}$.

The second statement follows from the fact that the canonical $\operatorname{spin}^{c}$ structure and its dual are the only ones with non-trivial SW invariant for any minimal complex surface of general type.

The case with $[\Sigma]^{2}=2 g-2$ is not covered in the corollary above, even though one can show for such a case that $\widetilde{X}$ may still have a SW basic class and indeed a symplectic structure on it. An example is given in Wang [25] where $\Sigma$ is taken to be a real part of an anti-holomorphic involution on a complex surface $X$. It would be interesting to determine in the example of [25], whether $\widehat{K}=p^{*} K \otimes P D[\widetilde{\Sigma}]$ can still be a SW basic class on $\widetilde{X}$ for the canonical bundle $K$ of $X$. 


\section{A final remark.}

Theorems 6.8 and 7.14 may be interpreted in terms of varying the holonomy of singular SW solutions on $\widetilde{X}$ along the surface $\widetilde{\Sigma}$. In fact it is possible to use this approach to prove alternatively the theorems, which is a little more complicated than our current proofs. Schematically it goes as follows: We pull back SW solutions from $\xi$ to $\breve{\xi}$, where $\breve{\xi}=p^{*}\left(\left.\xi\right|_{Y^{\prime}}\right)$ is a spin ${ }^{c}$ structure on $Y^{\prime}=X \backslash \Sigma$. The point is that we can choose carefully a Lipschitz Riemann metric on $\widetilde{X}$ [23] so that these solutions on $\breve{\xi}$ can be viewed as being defined everywhere on $\widetilde{X}$ with holonomy 0 . (The removable singularity theorem does not hold here as our metric is not smooth but only Lipschitz.) The SW invariants of $\xi$ and $\widetilde{\xi}$ are the same modulo 2 using the virtual neighborhood method in Section 2 . Because the determinant bundles of $\breve{\xi}$ and $\widehat{\xi}$, namely $\widetilde{L}$ and $\widehat{L}$, differ by a twist of $P D[\widetilde{\Sigma}]^{-1}$, we are suggested to consider a family of SW moduli spaces on $\breve{\xi}$ with the holonomy parameter interpolating between 0 and 1 , as in the Donaldson invariant case according to Kronheimer and Mrowka [11]. With the help of Theorem 7.11, we can get the required excision results as the holonomy approaches 0 and 1 , which in turn give the relation between the SW invariants of $\breve{\xi}$ and $\widehat{\xi}$. What is interesting about this approach is that unlike the Kronheimer-Mrowka picture, it does realize the interpolation without contradiction. This is possible precisely because the adjunction inequality is satisfied here!

Acknowledgments. We would like to thank Tom Mrowka and Peter Ozsvath for explaining their recent work; parts of Section 7 arose from our discussions with Peter Ozsvath. During the course of writing the paper, we benefited a great deal from several examples pointed out by Selman Akbulut and Ron Fintushel. Finally we are grateful to the referees for constructive suggestions and improvements.

\section{References.}

[1] S. Akbulut and R. Kirby, Branched covers of surfaces in 4-marifolds, Math. Ann. 252 (1980), 111-131.

[2] S. Cappell and J. Shaneson, Some new four-manifolds, Ann. Math. 104 (1976), 61-72.

[3] R. Fintushel and R. Stern, An exotic free involution on $S^{4}$, Ann. Math. 113 (1981), 357-365. 
[4] R. Fintushel and R. Stern, Pseudofree orbifolds, Ann. Math. 122 (1971), 335364.

[5] R. Fintushel and R. Stern, Knots, links, and 4-manifolds, Invt. Math. 134 (1998), 363-400.

[6] I. Hambleton and R. Lee, Perturbations of equivariant moduli spaces, Math. Ann. 293 (1993), 17-37.

[7] F. Hirzebruch, The signature of ramified coverings, Global analysis in honor of K. Kodaira, D. Spencer and S. Iyanage eds. Academic Press (1969).

[8] N. Hitchin, Harmonic spinors, Adv. Math. 14 (1974), 1-55.

[9] D. Kotschick, On irreducible 4-manifolds, preprint 1995.

[10] D. Kotschick, J. Morgan, and C. Taubes, Four-manifolds without symplectic structures but with non-trivial Seiberg-Witten invariants, Math. Research Letters, 2 (1995), 119-124.

[11] P. Kronheimer and T. Mrowka, Gauge theory for embedded surfaces I, Topology, 32 (1993), 773-826.

[12] P. Kronheimer and T. Mrowka, Gauge theory for embedded surfaces II, Topology 34 (1995), 37-97.

[13] P. Kronheimer and T. Mrowka, Embedded surfaces and the structure of Donaldson's polynomial invariants, J. Diff. Geom. 41 (1996), 573-734.

[14] P. Kronheimer and T. Mrowka, The genus of embedded surfaces in the project plane, Math. Res. Letters 1 (1994), 797-808.

[15] J. Morgan, Z. Szabo, and C. Taubes, A product formula for the Seiberg-Witten invariants and the generalized Thom conjecture, J. Diff. Geom. 44 (1996), 706788.

[16] J. Morgan, T. Mrowka, and D. Ruberman, The $L^{2}$-moduli space and a vanishing theorem for Donaldson polynomial invariants, Vol. 1, Monographs in geometry and topology, eds. N. Hitchin, R. Kirby and J. Wolf. International Press Inc. 1994.

[17] T. Mrowka, P. Ozsvath, and B. Yu, The generalized Thom conjecture, preprint, 1996.

[18] T. Mrowka, P. Ozsvath, and B. Yu, Seiberg-Witten monopoles on Seifert fibered spaces, Comm. Analy. Geom. 5 (1997), 685-791.

[19] P. Ozsvath and Z. Szabo, The symplectic Thom conjecture, to appear in Ann. Math. 
[20] Y. Ruan, Symplectic topology and extremal rays, Geom. Funct. Anal. 3 (1993), 395-430.

[21] Y. Ruan, Virtual neighborhoods and monopole equations, Proceedings of the First International Press Lecture Series at Irvine, California. Edited by $R$. Stern. International Press, 1998.

[22] Z. Szabo, Simply-connected irreducible 4-manifolds with no symplectic structures, Invt. Math. 132 (1998), 457-466.

[23] S. Wang, Moduli spaces over manifolds with involutions, Math. Ann. 296 (1993), 119-138.

[24] S. Wang, A vanishing theorem for Seiberg-Witten invariants, Math. Res. Letters 2 (1995), 305-311.

[25] S. Wang, Branched covers along real parts, Proc. Amer. Math. Soc. 125 (1997), 2803-2808.

[26] E. Witten, Monopoles and four-manifolds, Math. Res. Letters 1 (1994), 769796.

UNIVERSITY OF WISCONSIN,

MADISON, WI 53706, USA

AND

UNIVERSITY OF MISSOURI,

Columbia, MO 65211, USA

E-mail addresses: ruan@math.wisc.edu

sw@math.missouri.edu

RECeIVed February 18, 1998. 